Review Article

\title{
Complementary and Alternative Medicine for Threatened Miscarriage: Advantages and Risks
}

\author{
Lingjing Lu, ${ }^{1}$ Juan $\mathrm{Li}^{1,2}{ }^{1,2}$ Yu Zhou, ${ }^{1}$ Hongxia $\mathrm{Ma}^{1,2}$ and Min $\mathrm{Hu} \mathbb{D I D}^{1,2}$ \\ ${ }^{1}$ Department of Traditional Chinese Medicine, The First Affiliated Hospital of Guangzhou Medical University, Guangzhou, \\ Guangdong, China \\ ${ }^{2}$ Institute of Integration of Traditional Chinese Medicine and Western Medicine, Guangzhou Medical University, Guangzhou, \\ Guangdong, China \\ Correspondence should be addressed to Min Hu; kindhumin@163.com
}

Received 6 January 2021; Revised 5 March 2021; Accepted 9 March 2021; Published 22 March 2021

Academic Editor: Yong Wang

Copyright (๑ 2021 Lingjing Lu et al. This is an open access article distributed under the Creative Commons Attribution License, which permits unrestricted use, distribution, and reproduction in any medium, provided the original work is properly cited.

\begin{abstract}
Threatened miscarriage is one of the most common complications causing pregnancy loss, and it affects approximately $20 \%$ of confirmed pregnancies. More and more women are seeking treatment with complementary and alternative medicine (CAM) for this common complication, and it has been reported that women have had successful pregnancies after threatened miscarriage when being treated with CAM, which mainly includes Chinese herbal medicines, acupuncture, and nutritional supplements as well as psychological interventions and other approaches. However, many experts are concerned about the safety and adverse events of certain CAM approaches in women with threatened miscarriage. Therefore, this review focuses on the status of CAM for threatened miscarriage and presents the potential therapeutic efficacy and safety of CAM based on some clinical and experimental studies. We thus hope to provide some instructive suggestions for the application of CAM for treating threatened miscarriage in the future.
\end{abstract}

\section{Introduction}

Threatened miscarriage is one of the most common pregnancy complications and is indicated by vaginal bleeding before the 20th week of gestation. It affects up to $20 \%$ of confirmed pregnancies, and approximately $50 \%$ of cases end in pregnancy loss [1-3]. Even if the miscarriage is avoided, women who suffer from threatened miscarriage remain at high risk for adverse pregnancy outcomes such as premature birth, antepartum hemorrhage, low birth weight, and neonatal death, as well as psychological anomalies [4-8], and thus threatened miscarriage is a physically and psychologically traumatic experience for women and their families. Many factors cause threatened miscarriage, including chromosomal defects, immunological dysfunction, maternal thrombophilic disorders, endocrine abnormalities, and uterine structural anomalies. Additionally, maternal age, previous miscarriage, environmental pollutants, infectious agents, and previous clinical interventions also contribute to a high risk of miscarriage [9-12], and the thorough evaluation of the intrauterine condition with sonography and maternal serum markers might help to diagnose threatened miscarriage or predict subsequent pregnancy outcomes [13]. Bed rest does not significantly reduce the risk of miscarriage, and progesterone and human chorionic gonadotropin (hCG) are most commonly prescribed in women with threatened miscarriage even though little evidence supports their effectiveness [11-13]. Rh prophylaxes like heparin plus aspirin and other regimens such as buphenine hydrochloride have been suggested to be beneficial for threatened miscarriage, but they are associated with adverse events and their effects have not been verified (Figure 1).

The use of complementary and alternative medicine (CAM) for promoting health and treating ailments is an increasing trend worldwide. Studies have reported that women use CAM more than men, and over $80 \%$ of women in the UK, $50 \%$ of women in Australia, $90 \%$ of women in Canada, nearly 25\% of women in Denmark, and nearly 50\% 
Threatened miscarriage

What causes it?

(i) Chromosomal abnormalities

(ii) Immunological dysfunction

(iii) Endocrine factors, such as poorly controlled diabetes, polycystic ovary syndrome, and thyroid disease

(iv) Physical problems with the uterus or cervix

(v) Infection with bacterial, viral, parasitic, fungal, or sexually transmitted diseases

\section{What are the risk factors?}

(i) Previous miscarriages $\geq 2$

(ii) Age $>34$ years

(iii) Smoking or drinking

(iv) Using cocaine or illegal drugs

(v) History of in vitro fertilization, embryo transfer, or artificial insemination

(vi) Environmental toxins

(vii) Low levels of folic acid

(viii) Certain antibiotics

Figure 1: Possible etiology and current management of threatened miscarriage.

of women in the USA are users of CAM [8, 14-17]. Pregnant women use CAM at similar rates to those of nonpregnant women, and health professionals are increasingly recommending that pregnant women use CAM [18-20]. A systematic review found that women commonly use CAM alone or in combination with other approaches during pregnancy, with $5.8-74.2 \%$ of pregnant women taking herbal or natural treatments and $12-95 \%$ of pregnant women using vitamins, with most of them using CAM during the first trimester [19]. The women in the reviewed studies assumed that CAM can promote maternal and fetal health, enhance the efficacy of western medicines, and relieve specific pregnancy-related conditions, but there is poor evidence to support such effects of CAM. In line with this, a series of studies have been performed regarding the safety of CAM for pregnant women $[17,21-23]$. To date, several studies have reported on women who have had successful pregnancies after threatened miscarriage when being treated with CAM, mainly including Chinese herbal medicine (CHM), acupuncture, nutritional supplements, and psychological interventions $[8,10,11,24]$. This review briefly summarizes the current progress in preventing and treating threatened miscarriage with diverse forms of CAM and discusses the potential risks of such treatments.

\section{CHM in Threatened Miscarriage}

The prevalence of herbal medicine use by pregnant women ranges between $7 \%$ and $79.9 \%$ depending on different geographic, social, cultural, and ethnic factors $[21,22,25,26]$. CHM has a history of about 5000 years, and it has become one of the main therapies in East Asia and has spread to western countries. CHM is widely accepted as an alternative form of medicine, and it is considered beneficial for preventing and treating miscarriage with fewer adverse events compared to current conventional western medicine $[10,27,28]$. There have been a number of cases of treating threatened miscarriage using $\mathrm{CHM}$ according to the theory of traditional Chinese medicine (TCM). However, the safety and side effects of using certain herbal medicines during pregnancy is still a matter of debate, and here we review the efficiency and safety of CHM in the treatment of threatened miscarriage.
2.1. Efficiency of CHM for Threatened Miscarriage. Most of the literature regarding $\mathrm{CHM}$ use during pregnancy has focused on miscarriage, and the majority of the publications are in Chinese $[10,28,29]$. It has been suggested that the effectiveness of CHM ranges from $79.22 \%$ to $100 \%$ in the treatment of threatened miscarriage according to randomized and semirandomized trials comparing CHM, conventional western medicine, and combinations of the two (Table 1). However, there is a lack of well-designed placebocontrolled randomized clinical trials. A meta-analysis of 44 randomized clinical trials indicated that $\mathrm{CHM}$ alone has a similar efficacy to that of conventional western medicines alone in the treatment of threatened miscarriage, and CHM in combination with other pharmaceuticals was found to be superior to pharmaceuticals alone in maintaining pregnancy after 28 gestational weeks in women with threatened miscarriage [28]. CHM alone or combined with conventional western medicines has also been shown to be more beneficial in improving vaginal bleeding, lower back pain, and abdominal pain [30]. Moreover, a majority of randomized clinical trials and some systematic reviews have demonstrated that a combination of CHM and other pharmaceuticals or psychotherapy might maintain pregnancy and increase the live birth rate for women with recurrent miscarriage $[29,31]$. These studies all suggest that CHM has the potential to prevent threatened miscarriage in subsequent pregnancies, but most studies have neglected to investigate the duration, follow-up, adverse events, or toxicity of CHM, and the sample sizes of the interventions have been small.

\subsection{Common Formulas and Single CHM for Threatened} Miscarriage. A CHM formula contains one or more compositions of herbal raw material and can be available in different preparations such as decoctions, pills, powders, and extracts in order to obtain optimized efficiency. At present, the most commonly used classic prescription for threatened miscarriage is Shoutai Pill [10], which was first described in the book Integrating Chinese and Western Medicine from the late Qing dynasty around 200 years ago. The Shoutai Pill is composed of four ingredients, with Herba Taxilli, Semen Cuscutae, and Radix Dipsaci replenishing the liver and kidney and strengthening the bones and muscles to maintain 


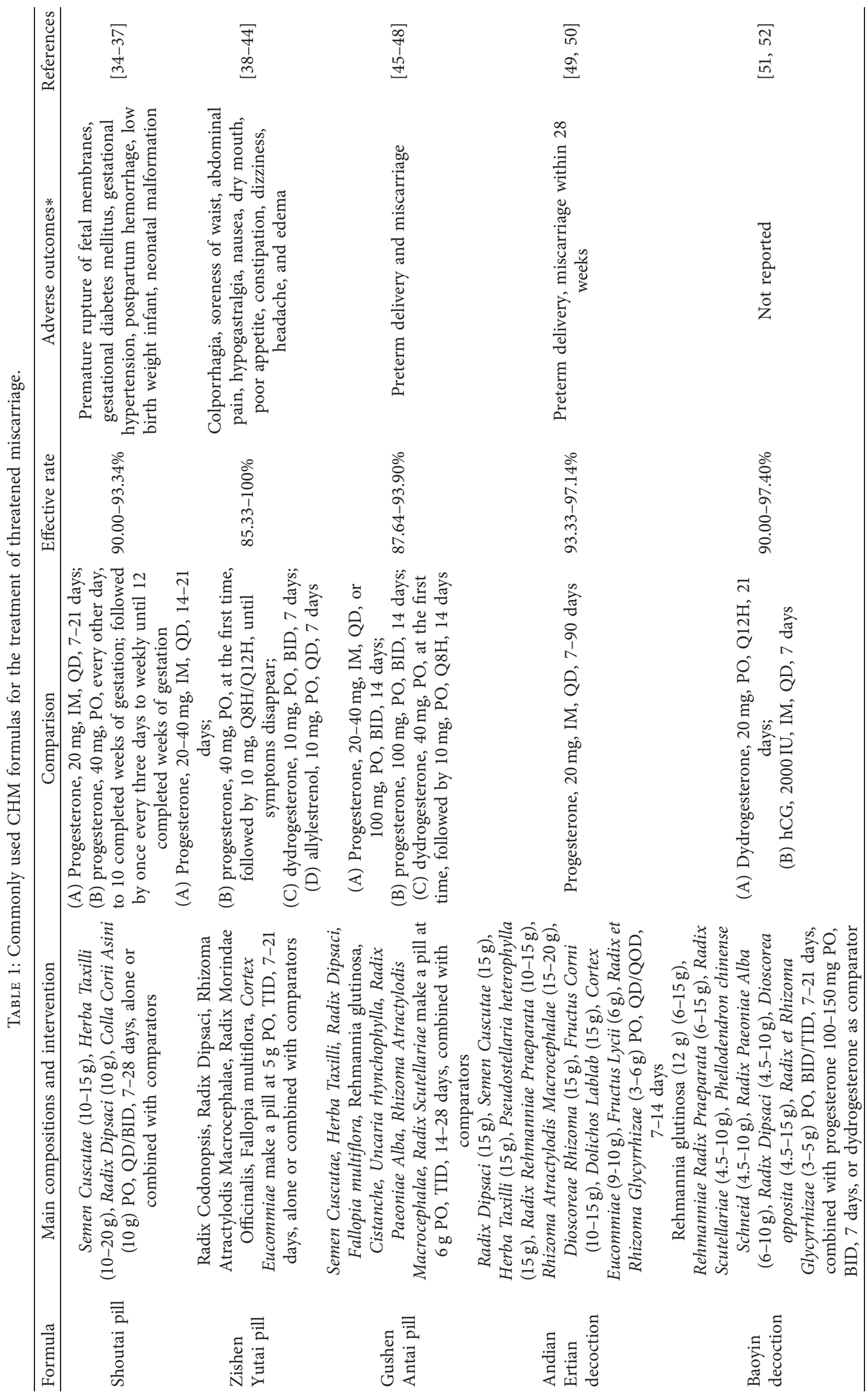




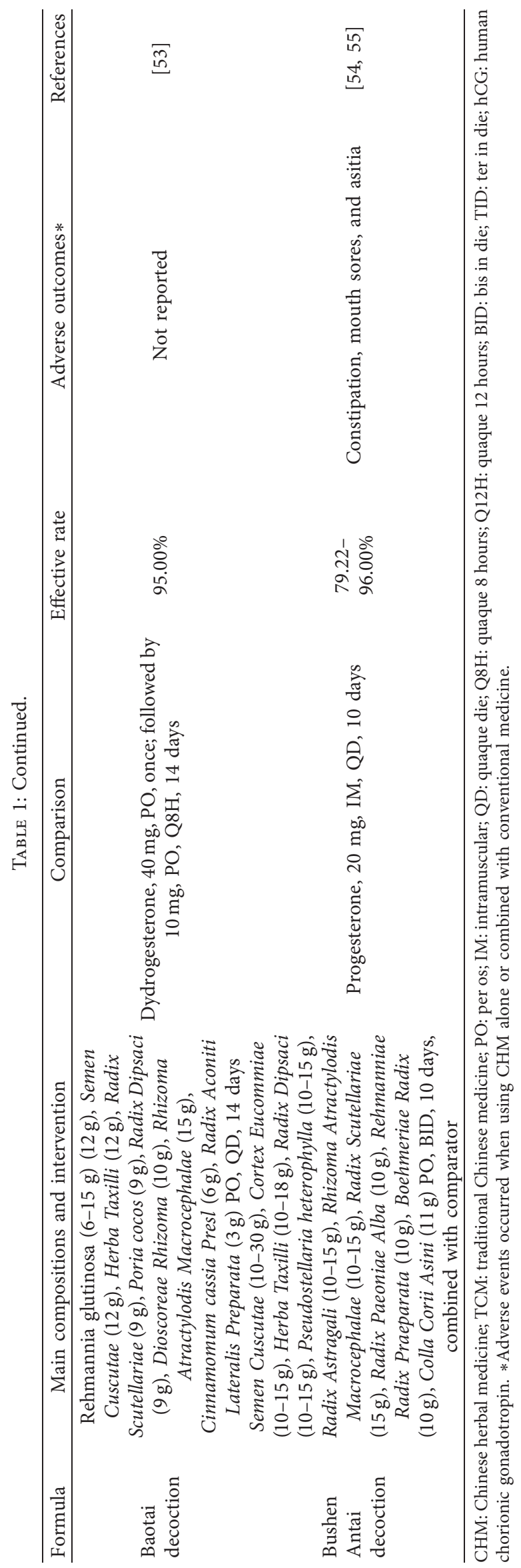


pregnancy and Colla Corii Asini nourishing the yin and blood and further strengthening Qi [32]. The four ingredients are documented in the Chinese Pharmacopeia and have been approved by the World Health Organization (WHO) for use in clinical trials. The prescription used alone or in combination with western medicines has been shown to be superior to western medicine alone for preventing miscarriage in the first trimester of pregnancy, and no adverse events have been reported [33-37] (Table 1).

On the basis of the Shoutai Pill, professional practitioners have further developed the recipe according to individualized clinical presentations for threatened miscarriage, for example, the Bushen Antai decoction. In addition, the generally applied prescriptions for threatened miscarriage include Baotai decoction, Zishen Yutai Pill, and other recipes that have been shown to be effective. With regard to the theory of TCM, these regimes are considered to regulate Qi and the blood and to improve kidney and spleen function in order to maintain pregnancy. We summarize the studies on the application of common CHM formulas for threatened miscarriage in Table 1 [38-55].

According to TCM theory, threatened miscarriage is due to insufficiency of the spleen and kidney, deficiency of Qi and blood, or both stasis and heat based on syndrome differentiation. Thus, herbal medicines that are frequently used to prevent miscarriage include those that tonify the kidney (e.g., Semen Cuscutae, Herba Taxilli, and Radix Dipsaci), those that tonify Qi and the blood (e.g., Colla Corii Asini and Codonopsis pilosula), those that nourish Qi to invigorate the spleen (e.g., Radix Astragali and Rhizoma Atractylodis Macrocephalae), and those that clear heat and cool the blood (e.g., Radix Scutellariae). Importantly, the proper concerted application of individual CHMs can have synergistic effects. The most commonly used combination is Semen Cuscutae and Herba Taxilli, which are contained in most of the formulas described above and can strengthen the kidney to prevent miscarriage. The most commonly used single compositions of CHM in formulas used to treat threatened miscarriage and prevent further pregnant complications are listed in Table 2 [10, 17, 56-77].

\subsection{Potential Mechanism of CHM for Threatened Miscarriage.} To explore the molecular mechanism of CHM in threatened miscarriage, many studies have been performed in humans and in diverse animal models both in vivo and in vitro. First, CHM might improve uterine function to retain pregnancy, and it has been reported that Shoutai Pill may reduce the miscarriage rate, increase serum progesterone and estrogen levels $[33,78]$, enhance endometrial thickness, and regulate the hemodynamic parameters of the uterine spiral artery in order to improve endometrial receptivity and promote implantation [79]. Second, CHM potentially contributes to the development of trophoblasts and reduces the miscarriage rate. For example, Shoutai Pill can regulate the bioactivity behavior of trophoblasts to prevent spontaneous miscarriage by regulating trophoblast proliferation, invasion, and $\mathrm{mi}-$ gration capacity, $\beta$-hCG secretion by trophoblasts, and apoptosis of trophoblasts in vitro [31, 80]. Furthermore,
CHM has the effect of regulating immunological function and anti-inflammatory and antioxidation activities, which is consistent with TCM theory on tonifying the kidney and preventing miscarriage $[79,81,82]$. Shoutai Pill can improve pregnancy outcomes by reducing placental damage and regulating oxidative stress, and Shoutai Pill increases serum IL-2 and IL-6 levels and placental glutathione and superoxide dismutase (SOD) levels and decreases 3,4-methylenedioxyamphetamine and reactive oxygen species (ROS) levels [82]. Another study found that CHM can treat threatened miscarriage by regulating the expression of the inflammatory factors IFN- $\gamma$ and IL-10 [83]. Overall, the application of CHM may improve endometrial function, inhibit uterine contraction, regulate immune response, and promote the development of the embryo. However, there are limited numbers of studies into the mechanisms through which individual CHMs or combinations have an effect on threatened miscarriage, and few of the reports are in English.

2.4. Safety and Adverse Events of CHM for Threatened Miscarriage. Almost half of pregnant women in the world use CHMs or other herbal medicines to support their pregnancy, to ameliorate disturbing symptoms and complications, and to reduce the need for western medication, and they widely consider that these treatments are safe and effective. However, all regimes, including CHM, have the potential risk for pregnancy and fetal development, but only a few studies have evaluated the safety of these interventions. CHMs are commonly used in threatened miscarriage in order to prevent pregnancy loss and further complications, and most clinical trials have reported no adverse events or significant risks. Some systematic reviews have concluded, however, that no data are available to demonstrate the safety of CHM for the mother or the infant $[29,31]$. In turn, a few studies have found adverse outcomes in relation to $\mathrm{CHM}$, including gastrointestinal reactions, preterm birth, premature rupture of membranes, stillbirth, asphyxia, and infections that cause neonatal death [27]. None of these clinical studies further explained the reasons for these adverse events, and there are limited data available for identifying the risks of CHM. A systematic review and meta-analysis indicated that CHM combined with conventional medicines had lower intervention failures for women with threatened miscarriage, and no obvious differences were found between the combination of CHM and conventional medicine and $\mathrm{CHM}$ alone regarding adverse events and toxicity in terms of pregnancy and perinatal outcomes [27]. In the pooled randomized controlled trials, $3.1-22.3 \%$ had intervention failure; $2-10 \%$ had dry mouth, constipation, and insomnia; $3 \%$ had diabetic complications; $5 \%$ had preterm delivery; and $1.8 \%$ had neurodevelopmental morbidity. Notably, some individual herbal medicines were reported to have potent risks during pregnancy; for example, a Danish prospective cohort study suggested that licorice causes an increase in blood pressure in pregnant women [17] (Table 2).

Moreover, various animal studies have indicated reproductive toxicity with the frequently used CHMs for threatened miscarriage, including fetal resorption, growth 


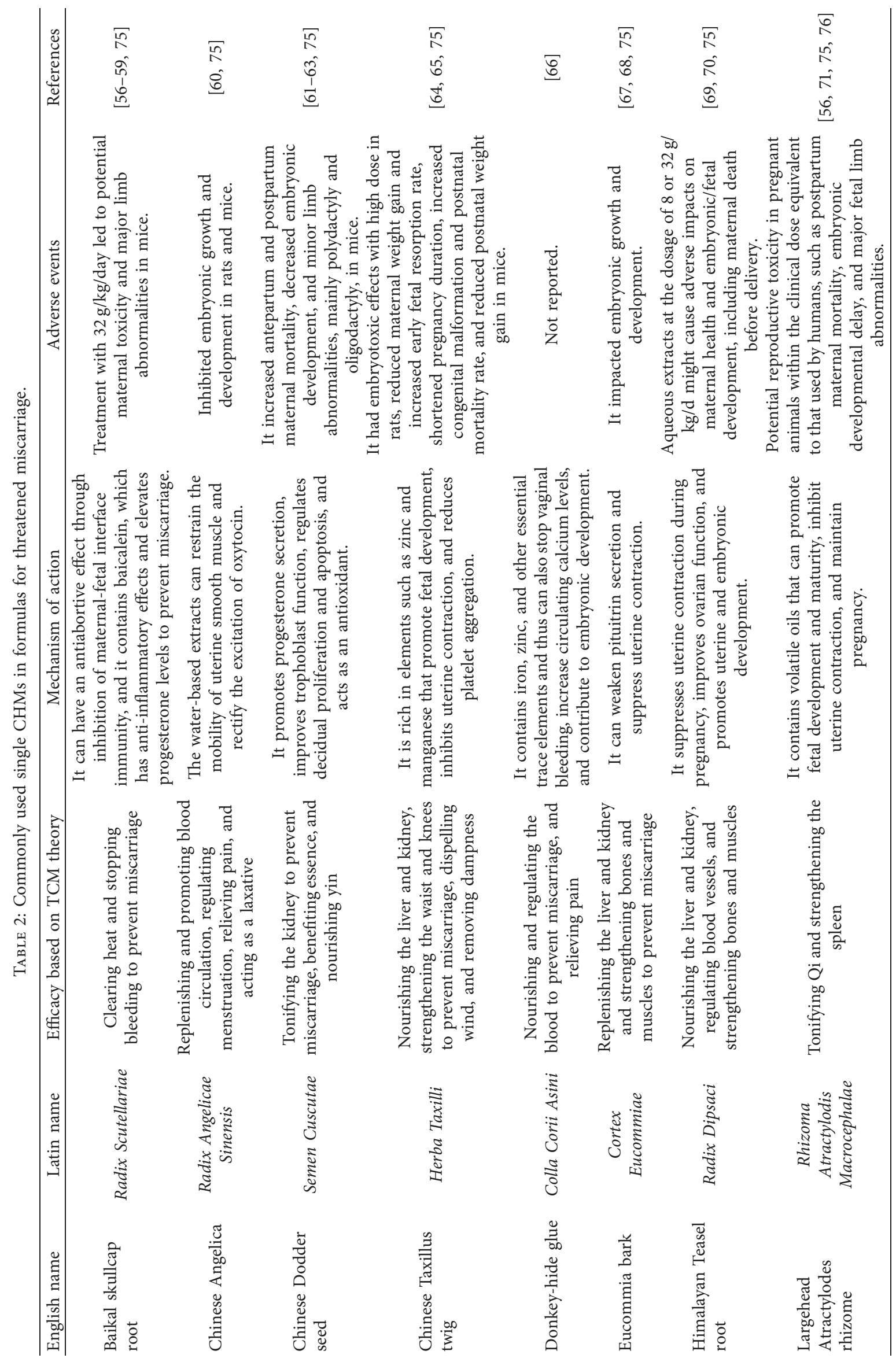




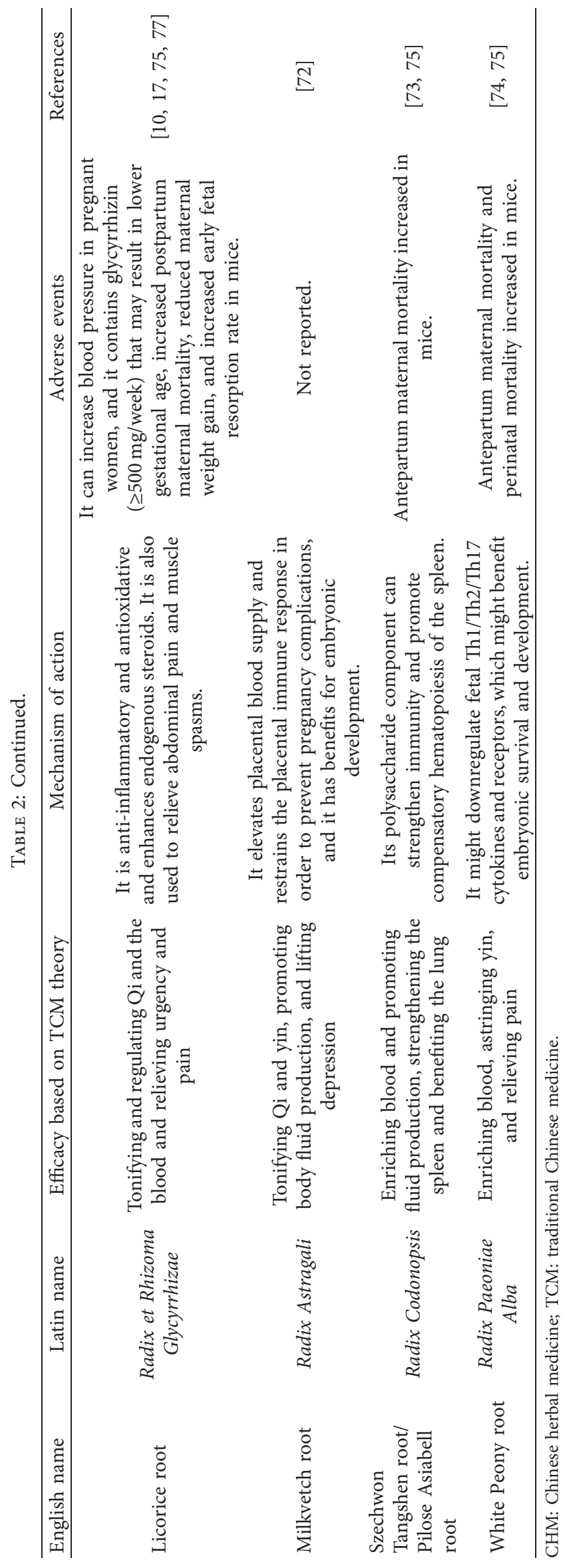


restriction, and congenital malformations [27]. For example, Wang et al. found 17 individual CHM extracts that seemed to be toxic to embryos and fetuses in pregnant mice [75]. Li et al. investigated the reproductive toxicity of Largehead Atractylodes Rhizoma in mice, rats, and rabbits [76], and the single composition increased prenatal and postnatal mortality and at high doses increased congenital malformations. Although these studies indicated that CHMs can have reproductive toxicity during pregnancy, the evidence is not sufficient. The experiments were conducted in normal pregnant animals rather than miscarriage models, and thus the data are not reliable sources for deriving pregnancy risks in humans for the tested CHMs, and this might explain why the results are in stark contrast to the outcomes after treatment in humans [84]. In addition, large doses of astragaloside, the main component of Astragalus membranaceus, can induce fetal toxicity, and Eucommia ulmoides Oliv. and total glucosides from peonies may lead to gene mutations and are suspected to be teratogenic $[74,85,86]$. In practice, TCM practitioners generally utilize CHM formulas in the treatment of threatened miscarriage, not single crude $\mathrm{CHM}$ extracts like those used in the animal experiments. For example, Zishen Yutai Pill, which is a common CHM to treat threatened miscarriage, shows no toxicity in the perinatal period in pregnant rats [87]. CHM synergies based on TCM theory might reduce the toxic effects, and there is a close correlation between the safety and efficacy of CHMs and the quality of the source materials used in their production.

So far, there is limited evidence for adverse events when using CHM to treat threatened miscarriage. Most CHMs are in a similar situation to that of the majority of pharmaceuticals available today, and neither their safety nor their risks during pregnancy have been verified. The current evidence from the evaluation of the safety of CHM in clinical use is valuable, but large-scale randomized placebo-controlled trials showing an unremarkable impact of pregnancy are warranted.

\section{Acupuncture and Moxibustion in Threatened Miscarriage}

3.1. The Role of Acupuncture in the Treatment of Threatened Miscarriage. Acupuncture and moxibustion are recognized nonpharmacological and alternative approaches, and they are increasingly used for reproductive conditions worldwide $[88,89]$. Based on the theory of TCM, together these treatments have the function of dredging the meridian and stimulating the energy response of the Qi and blood, thereby strengthening the body's resistance to disease and eliminating pathogenic factors. Traditional acupuncture has specific theories relating to promoting optimal early pregnancy responses, and it is recommended as a treatment modality for threatened miscarriage in acupuncture texts. Although the therapies exist in textbooks, there is yet no quality research to support the use of acupuncture for threatened miscarriage. Within fertility research, acupuncture has been shown to improve hormonal responses with decreased miscarriage rates, thus raising the possibility that acupuncture might promote specific beneficial effects in early pregnancy $[90,91]$. Acupuncture might thus be a potential alternative option for threatened miscarriage under proper treatment control [11].

The safety of acupuncture in pregnancy is reasonably well accepted, but there is still a lack of high-quality scientific research to validate the safety and effectiveness of acupuncture for threatened miscarriage [92]. Many experts have raised concerns about the safety of acupuncture for treating women in early pregnancy $[93,94]$, and there remains a debate regarding the needling points that are historically considered to be forbidden during pregnancy, like SP6, LI4, BL60, BL67, GB21, and LU7. Nevertheless, electroacupuncture at these forbidden points did not aggravate the miscarriage rate or fetal loss over the course of gestation in pregnant rats [95]. Furthermore, there is no reliable evidence to suggest that acupuncture can induce miscarriage or premature delivery, and the adverse events with the use of forbidden points during pregnancy were similar to the control interventions [95]. The incidence of adverse events probably related to acupuncture during pregnancy was $1.3 \%$ according to a systematic review, and most of these were mild adverse events such as needling pain. Severe adverse events and fetal complications due to preterm delivery were rare, and the miscarriage rate was $5 \%$, which was lower than that for control interventions [96]. A recent retrospective cohort study in South Korea reported consistent observations as previous studies, and there were no significant differences in delivery outcomes between pregnancies in the acupuncture and control groups [97]. Even though many of these studies have had small sample sizes and the methodologies have had a high risk of bias, they consistently suggest the safety of acupuncture during pregnancy.

Nowadays, about $4 \%-13 \%$ of pregnant women in Europe receive acupuncture for pregnancy and childbirth issues, and most treatments are given in the first trimester $[98,99]$. Acupuncture has been shown to reduce the risk of miscarriage by increasing blood flow to the uterine lining and by aiding implantation, and thus it has potential for the treatment of threatened miscarriage. Professional practitioners are more and more using acupuncture during pregnancy, and surveys on acupuncture use conducted in the UK, Australia, and New Zealand showed that over a half of the acupuncturists had treated women for threatened miscarriages [91]. In a randomized feasibility trial with semistructured participant interviews with 40 women with threatened miscarriage, the participants received a pragmatic acupuncture and moxa protocol or touch intervention with medical self-care advice. The women who received acupuncture on the basis of TCM theory reported reduced vaginal bleeding, cramping, and back pain. These findings demonstrated that acupuncture was a feasible and pragmatic intervention that may be effective for women experiencing threatened miscarriage [92] (Table 3). However, although the application of a pragmatic acupuncture treatment protocol allowed flexibility for diagnosis and treatment, reflecting a treatment approach applicable to the real world, the protocol is difficult to use because the protocol designs do not provide information regarding the specific acupuncture points or needling effects. In addition, it was 


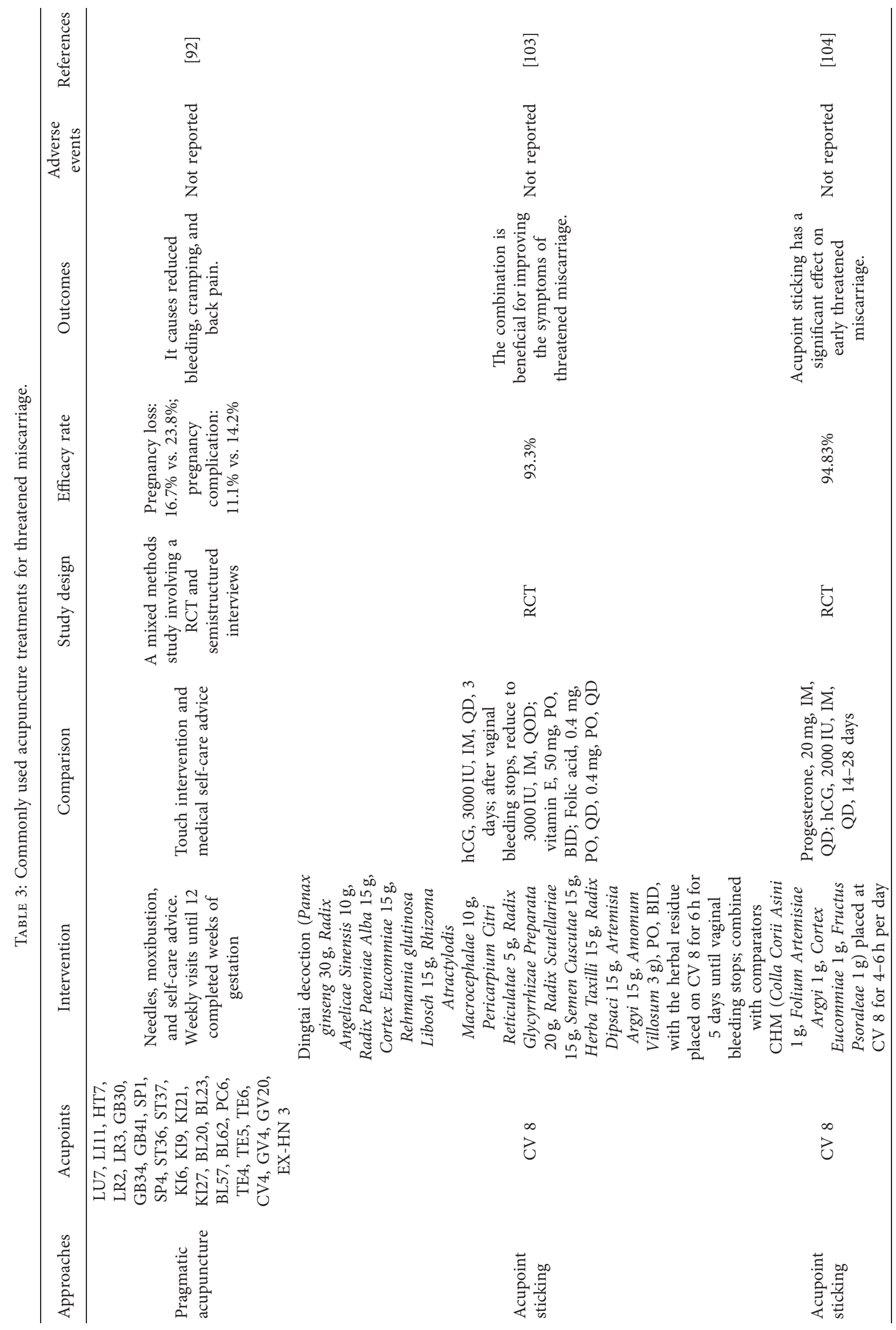




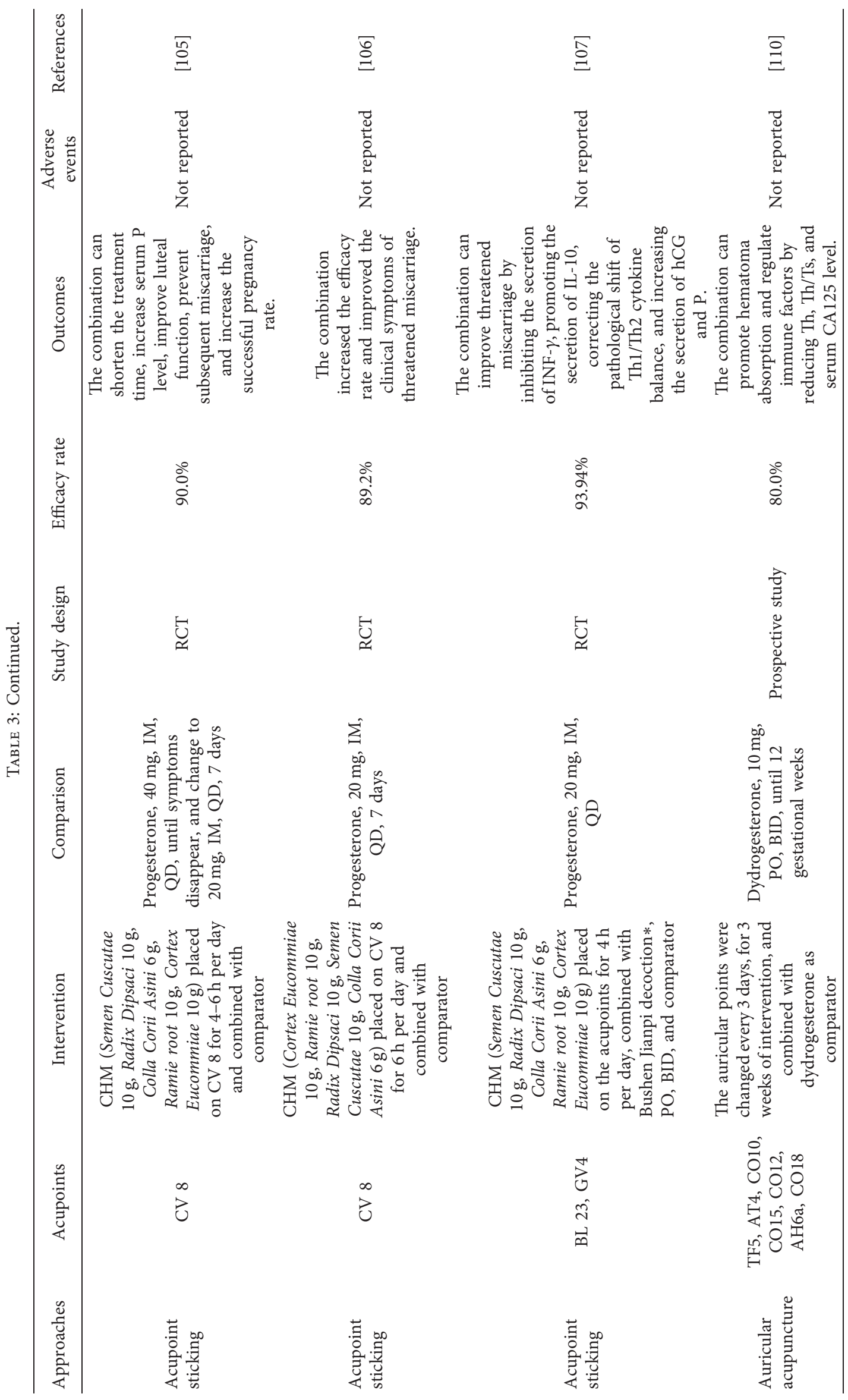




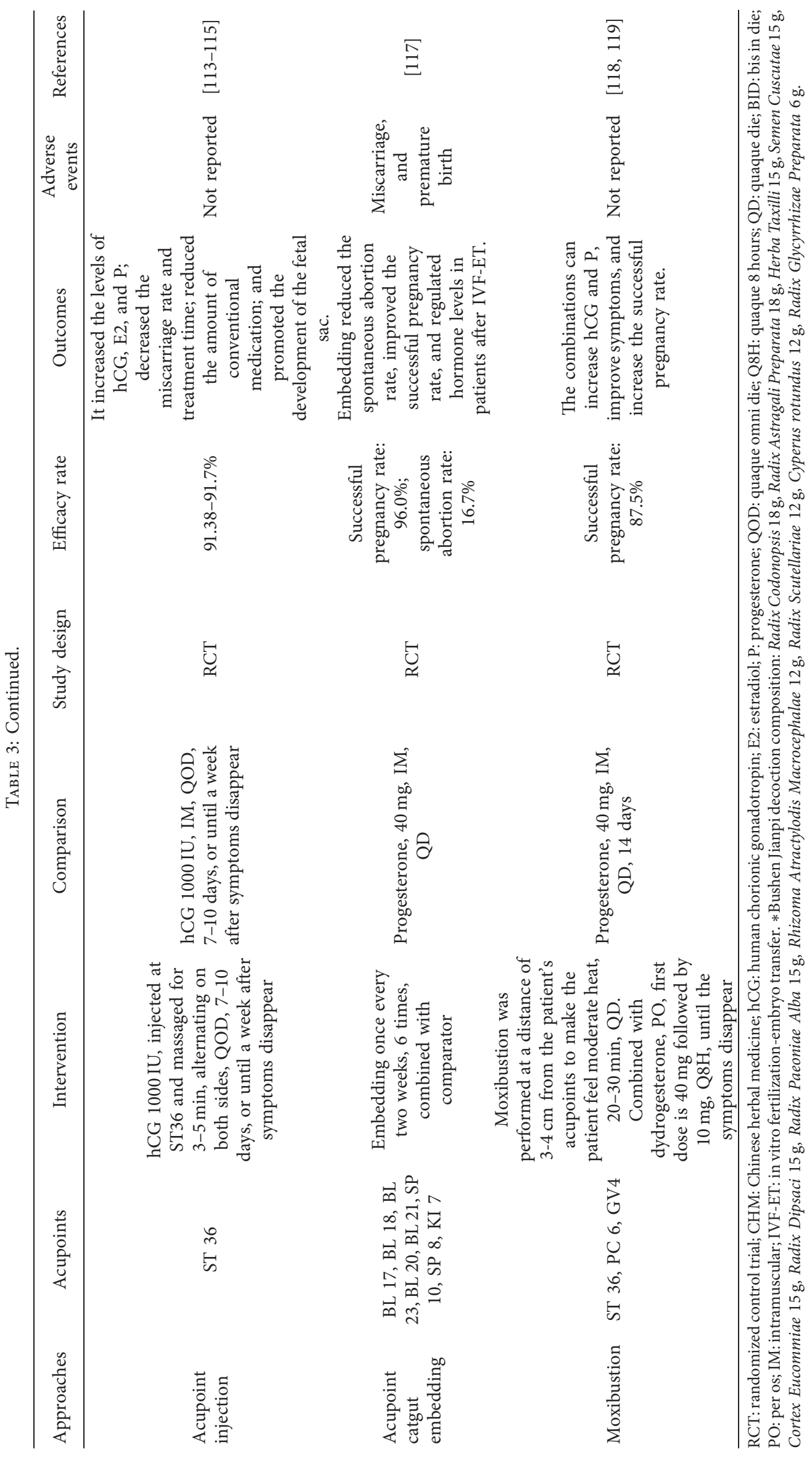


reported that different styles of acupuncture treatment had benefits for threatened miscarriage, such as auricular point acupuncture, acupoint injection, acupoint sticking, and catgut embedding $[11,100]$. Acupuncture might be a safe therapeutic approach for threatened miscarriage, but the current studies are of poor quality and are usually written in Chinese, and thus further research is required to explore whether acupuncture can reduce the incidence of miscarriage.

\subsection{Different Styles of Acupuncture in the Treatment of Threatened Miscarriage}

3.2.1. Acupoint Sticking. Acupoint sticking is a noninvasive therapy based on TCM theory in which pastes of various medicinal extract mixtures are placed on the skin at specific acupoints or at the diseased sites. This achieves effects not only through the activities of the drugs, but also by activating acupoints and meridians. Acupoint sticking is generally applied in common chronic diseases, particularly for pain relief [101]. The approach is acceptable for patients and is flexible and safe, and thus a large number of clinical practitioners use the intervention to treat women with threatened miscarriage in China. A meta-analysis investigated the efficacy and safety of different styles of acupuncture in threatened miscarriage and demonstrated that acupoint sticking therapy is the best acupuncture approach for this common pregnancy complication [102]. Generally, acupoint sticking with pastes made from modified CHM formulas combined with oral administration of Chinese herbal decoctions and/or progesterone can reduce the miscarriage rate, and the effectiveness of such combinations has been shown to be better than conventional medicine alone [103-107] (Table 3). However, the efficacy and safety of these treatments are in urgent need of confirmation in future studies.

3.2.2. Auricular Acupuncture. Auricular acupuncture is a therapy based on the stimulation of specific points on the ear. Auricular acupoint therapy is applied for situations of pain, inflammatory diseases, functional disorders, and endocrine and metabolic disorders by regulating reticular formation and by regulating the sympathetic and parasympathetic nervous systems [108]. This intervention appears to have analogous effects on sedation as opioids and can reduce pain and anxiety [109]. Auricular acupuncture is usually applied as an adjuvant with conventional approaches for relieving pregnancy-related pain and complications. The acupoints on the ear might regulate pregnancy and nourish fetal developing by achieving chronic shallow acupressure. TCM practitioners reported that a combination of auricular acupuncture and dydrogesterone could promote hematoma absorption in women with threatened miscarriage in early pregnancy complicated with subchorionic hematoma, was more effective than dydrogesterone alone, and could regulate immune factors over a 3-week course of treatment [110] (Table 3).
3.2.3. Acupoint Injection. Acupoint injection, which is also called pharmacoacupuncture, aqua acupuncture, water acupuncture, or herbal acupuncture, is widely used in East Asia and is based on the same meridian theory of acupuncture. Acupoint injection therapy is the combination of acupuncture and medications and has been demonstrated to benefit patients with nonspecific chronic low back pain [111]. The injection of sterile solutions of Chinese herbal extracts or western medicines into acupoint locations leads to synergistic therapeutic effects of acupuncture, medicine, and meridian activation. The drugs are absorbed through the subcutaneous tissues and capillary vessels, while the needles give positive stimulation at local acupoints, which may promote local blood circulation, elevate metabolic ability, and ameliorate the pathology while promoting inflammation resolution [112]. Many studies have found that acupoint injection therapy has a significant effect on threatened miscarriage compared to western medicine alone in reducing early pregnancy loss. The most common therapy is injection of 1000 IU of hCG into the Zusanli acupoint (ST 36) every other day [102, 113-115] (Table 3). This method is safe and reliable, with little side effects, and it treats diseases through the combined action of drugs and acupoints. More well-designed studies are needed to determine the efficacy and safety of acupoint injection therapy for threatened miscarriage.

3.2.4. Acupoint Catgut Embedding. Acupoint catgut embedding refers to embedding absorbable catgut sutures into certain acupoints, and the continuous stimulation of acupoints is believed to cure diseases and strengthen the body. Catgut embedding at acupoints evolved from needle embedding at acupoints by replacing needles with catgut, and this not only has effects similar to standard acupuncture, but also has effects due to the prolonged stimulation time, which can reach 2 weeks or even longer. The approach showed a tendency for equal effects compared to other kinds of acupuncture for reducing abdominal obesity, but the approach had fewer reported adverse events [116]. Acupoint catgut embedding has also been indicated to prevent miscarriage in patients with threatened miscarriage. In a randomized control trial, the patients who had experienced in vitro fertilization-embryo transfer were treated with catgut embedding once every two weeks until 12 weeks of gestation. The early miscarriage rate was $16.7 \%$, and the pregnancy rate was $96.0 \%$; these were significantly lower and higher, respectively, compared to those who received progesterone [117] (Table 3). It thus seems that acupoint catgut embedding is beneficial for threatened miscarriage and is worth further investigation.

3.2.5. Moxibustion. Moxibustion consists in burning the leaves of Chinese mugwort (Artemisia vulgaris) close to the skin to induce heat at certain acupoints. The intention is to warm up and invigorate the flow of Qi while also eliminating various pathogenic influences on the body. This therapy is considered safe and has no side effects, making it a viable 
solution for pregnant women looking for a remedy for pregnancy-related symptoms. Two randomized, controlled studies in China looked at the combination of daily moxibustion and dydrogesterone compared to progesterone intramuscular injection or dydrogesterone oral administration. The moxa was placed over the acupoints Zusanli (ST36), Neiguan (P6), and Mingmen (GV4), and the combination approach was superior to progesterone or dydrogesterone alone in decreasing the miscarriage rate in women with threatened miscarriage $[118,119]$ (Table 3). In contrast, it has been reported that moxibustion can stimulate estrogen and prostaglandin production and increase fetal activity and uterine contractions in order to reduce noncephalic presentations at birth [120]. Thus, the safety and efficacy of moxibustion in the treatment of threatened miscarriage are still a matter of debate.

\section{Nutritional Supplements in Threatened Miscarriage}

Nutritional supplements, also known as dietary supplements, are used as an auxiliary way of supplying amino acids, trace elements, vitamins, and minerals for maintaining maternal health and fetal development. Dietary habits constitute important risk factors for potentially harmful nutritional deficiencies in pregnant women, and inadequate diet and nutritional supplementation during pregnancy can damage placental function and increase the risk of miscarriage and other pregnancy complications $[24,121,122]$. Nutritional supplements include essential nutrients to maintain human health, and vitamins and trace elements can directly affect the growth and development of the fetus. Mineral elements and vitamins can maintain the activity of the internal environment and various bioactive substances, and they participate in the body's energy transfer and metabolic regulation. Therefore, it is of great significance to give nutritional supplements before and during pregnancy because adequate nutritional support for pregnant women is essential for maintaining their own health and for supporting fetal development, growth, and future outcomes.

Oxidative stress can also lead to increased risk of threatened miscarriage, spontaneous abortion, recurrent pregnancy loss, and preeclampsia $[123,124]$. Antioxidant supplementation may be effective in controlling the production of ROS and combating damage caused by free radicals, and it continues to be explored as a potential strategy for overcoming pregnancy disorders associated with miscarriage [124]. Natural antioxidant compounds alone or in combination with other antioxidants or micronutrients during pregnancy may have the potential to prevent pregnancy loss and other complications [123]. Nutritional supplements and antioxidant intake are usually within the dietary reference intake range, which includes the effects of food and fortified foods, and only a few minor adverse events, such as gastrointestinal reactions, have been shown to occur [125]. Here, we review studies on the efficacy and safety of nutritional supplements for women with threatened miscarriages.
4.1. Essential Trace Elements. Essential trace elements are involved in various biochemical pathways, and they play a crucial role in maternal health and fetal growth and development during pregnancy $[126,127]$. Alterations in the concentrations or homeostasis of these micronutrients during pregnancy appear to be closely linked to various disorders and adverse pregnancy outcomes like miscarriage, preterm delivery, stillbirth, intrauterine growth restriction, fetal malformations, and premature rupture of membranes. Trace elements have been shown to facilitate various vital biochemical reactions by acting as cofactors for many enzymes and by stabilizing the structures of enzymes and proteins, and they are significant for all levels of cellular functions [128, 129]. It may be necessary to supplement essential trace elements in women with threatened miscarriage $[126,130]$.

Zinc $(Z n)$ is one of the most important essential trace elements in humans, and it participates in multiple biological functions including protein synthesis, cellular division, and nucleic acid metabolism [131]. Zn deficiency during pregnancy gives rise to the risk of pregnancy loss, preeclampsia, placental abruption, preterm birth, low birth weight, birth defects, circulatory disorders, immune response impairments, and psychological disorders [132-135]. Women with threatened miscarriage have been shown to have $35.7 \%$ lower $\mathrm{Zn}$ levels than healthy pregnant women $[126,136]$, and low serum $\mathrm{Zn}$ levels during the early weeks of pregnancy have been shown to lead to miscarriage and fetal congenital malformations by reducing cell proliferation and protein synthesis, which is associated with increased cellular oxidative damage and apoptosis [126, 137]. Also, it was shown that women with serum $\mathrm{Zn}$ levels below $10.5 \mu \mathrm{mol} / \mathrm{l}$ had a miscarriage rate of $23.5 \%$, and adding supplements reduced the rate to $2 \%$ [138]. However, studies of the effects of $\mathrm{Zn}$ supplementation during pregnancy have shown inconsistent results, possibly in part because of the challenges in establishing the baseline $\mathrm{Zn}$ status in different populations [139]. Zn supplementation at $30 \mathrm{mg}$ daily did not seem to confer any benefit on infants' mental development among poor women in Bangladesh [140]. Zn supplementation might be prudent for women with poor gastrointestinal function, but the evidence was limited for beneficial effects of general $\mathrm{Zn}$ supplementation during pregnancy, and such treatment should be considered with caution.

Copper $(\mathrm{Cu})$ as a trace element plays an important role in the maturation of hematopoietic cells in normal pregnancy and embryogenesis and in fetal and postnatal growth, and low plasma $\mathrm{Cu}$ levels have been found in spontaneous, threatened, and missed miscarriages during the first trimester of pregnancy [141]. Women with threatened miscarriage had $47.0 \%$ lower $\mathrm{Cu}$ levels than women with healthy pregnancies, and approximately $30 \%$ failed to reach term due to $\mathrm{Cu}$ deficiency $[136,142,143]$. There is a direct and positive correlation between $\mathrm{Zn}$ and $\mathrm{Cu}$ levels in women with threatened miscarriage, and there is a significant negative correlation between $\mathrm{Cu}$ and the ratio of $\mathrm{Cu}$ to $\mathrm{Zn}$ in women with a history of spontaneous abortion. Both $\mathrm{Zn}$ and $\mathrm{Cu}$ have a positive role in pregnancy outcomes, and optimum levels of $\mathrm{Zn}$ and $\mathrm{Cu}$ might be able to reduce the 
occurrence of spontaneous miscarriage $[133,136]$. Women who are $\mathrm{Cu}$ deficient usually take supplements of $1 \mathrm{mg}$ of $\mathrm{Cu}$ daily and $30 \mathrm{mg}$ of $\mathrm{Zn}$ separately for 14 days in order to normalize decreased SOD function [140]. In contrast, some studies found maternal serum $\mathrm{Cu}$ levels to be increased in threatened miscarriage compared with healthy pregnancy due to the increase of ceruloplasmin as a result of elevated levels of estrogen $[126,144-146]$. Thus, there is a debate as to whether women with threatened miscarriage should be treated with $\mathrm{Cu}$ supplements.

Iron $(\mathrm{Fe})$ is a component of hemoglobin and myoglobin, and it supports maternal erythropoietic expansion and fetal growth and development during pregnancy. It is also involved in the transport, storage, and use of oxygen [147]. Fe deficiency is associated with increased oxidative stress, placental and fetal hypoxia, and reduced immunity during pregnancy. Previous studies found that serum Fe levels were lower in cases of threatened miscarriage, and the serum Fe level might be an important diagnostic and prognostic parameter [126, 148]. Pregnancy loss is associated with profound changes in maternal Fe metabolism [149], and maternal $\mathrm{Fe}$ deficiency, which is common in pregnant women [150], negatively impacts the mother's health and fetal development and increases the risk of prenatal and postnatal complications. Foods rich in Fe include red meat, shellfish, eggs, beans, and leafy green vegetables, and high $\mathrm{Fe}$ intake with higher Fe bioavailability is needed by pregnant women to prevent adverse pregnancy outcomes and to meet the needs of the fetus. Pregnant women should routinely receive Fe supplements tailored according to serum ferritin levels. The International Nutritional Anemia Consultative Group recommends oral ferrous iron supplementation at $60 \mathrm{mg} /$ day during pregnancy to prevent Fe-deficiency anemia. Depending on the severity of anemia, international guidelines recommend elemental ferrous iron at $100-200 \mathrm{mg}$ daily or $60 \mathrm{mg}$ twice daily as the first-line treatment [151-153]. Prolonged-released ferrous sulfate (ferrous sulfate-polymeric complex) has the lowest incidence of adverse events of all the available supplements, and this has positive implications for compliance [121]. Moreover, the metabolism of essential trace elements is closely related. For example, Fe deficiency results in an increase in liver $\mathrm{Cu}$ levels [126], high intakes of supplemental Fe or the presence of any gastrointestinal disease can interfere with $\mathrm{Zn}$ absorption, and $\mathrm{Zn}$ and selenium supplementation need to be given along with Fe during pregnancy [154]. The available evidence suggests that Fe supplements are needed for pregnant women, but overtreatment should be avoided.

Magnesium $(\mathrm{Mg})$ and manganese $(\mathrm{Mn})$ are additional essential trace elements for metabolic regulation, and serum $\mathrm{Mg}$ and Mn levels have been reported to be lower in women with threatened miscarriage compared to those with a healthy pregnancy [126]. Mg deficiency is associated with hypertension, preeclampsia, placental dysfunction, and premature labor in pregnant women, but Mg levels have not been shown to be different between pathological pregnancies and healthy controls [155]. Furthermore, some studies have reported that $\mathrm{Mn}$ concentrations are increased in women with a history of miscarriage [156]. There is still limited evidence for the use of $\mathrm{Mg}$ and $\mathrm{Mn}$ supplements for the treatment of threatened miscarriage.

4.2. Vitamin Supplements. Vitamins are essential nutrients to maintain metabolism, physical growth, and development and to prevent disease. Nearly 30\% of pregnant women suffer from a vitamin deficiency, and about $75 \%$ of these would show a deficit of at least one vitamin in the absence of prophylaxis [157]. Vitamin supplementation during pregnancy may prevent adverse pregnancy outcomes and reduce the risk of pregnancy loss [24], but there are still contradicting findings in the research on vitamin supplements and the risk of miscarriage. Insufficient vitamin intake is related to an increased risk of miscarriage, and the intake of multivitamins with Fe and folic acid may decrease the risk of stillbirth, but single vitamin supplements either before or during early pregnancy have not been shown to reduce the miscarriage rate $[24,158,159]$.

Vitamin $A$ is a crucial micronutrient for pregnant women and their fetuses, and vitamin A can reduce the risk of anemia, infection, and night blindness in pregnant women [157, 160, 161]. Vitamin A deficiency is prevalent in developing countries, and it impairs $\mathrm{Fe}$ status and reduces resistance to infections [157]. In a systematic Cochrane review, there was no positive effect on the total risk of fetal loss, on early or late miscarriage, or on stillbirth in women who received vitamin A with any other combination [24]. Vitamin A supplements enhance infant birth weight and growth in HIV-infected women [157]. The recommended upper limit for vitamin A supplements is $3000 \mathrm{IU} /$ day, but when used in excess during the first trimester of pregnancy, such levels can have teratogenic effects on the first 60 days following conception [160]. However, there is no evidence of vitamin A supplements leading to congenital malformations or other adverse effects [24]. Further research is needed on the dose and duration of vitamin A supplements during pregnancy, especially its use for threatened miscarriage.

Vitamin $B$ complex plays a crucial role in maternal health and fetal development. Vitamin B facilitates the metabolism of homocysteine, and vitamins B6 and B12 determine the homocysteine concentration in the blood, while disturbances in maternal and fetal homocysteine metabolism may result in miscarriage, and hyperhomocysteinemia is considered a risk factor for recurrent miscarriage [162, 163]. Vitamin B6 deficiency is associated with miscarriage, preeclampsia, gestational carbohydrate intolerance, hyperemesis gravidarum, and neurological diseases in infants [157]. Supplementation with vitamin B6 has been shown to reduce the chance of miscarriage by $50 \%$ and to prevent stress from affecting fetal growth [164, 165]. Vitamin B6 has also been indicated to improve insulin resistance and to reduce oxidative DNA damage and C-reactive protein, and it may act similarly on progesterone and reduce prolactin in order to prevent miscarriage $[166,167]$. Vitamin B12 deficiency is associated with recurrent early pregnancy loss, and the miscarriage risk increases by 3.8 -fold with every quartile of severity in vitamin B12 deficiency $[168,169]$. Vitamin B12 supplementation has been shown to 
lead to successful pregnancy in $80 \%$ of women with vitamin B12 deficiency [170]. Deficiency in the conversion of vitamin B3 into nicotinamide adenine dinucleotide causes congenital malformations and miscarriages in mouse models, and vitamin B3 supplementation has been shown to prevent miscarriages and birth defects $[171,172]$. However, recent reviews of the literature indicate that there is insufficient evidence for the benefits and harms of routine vitamin $\mathrm{B}$ supplementation in humans for threatened miscarriage.

Folate, also known as vitamin B9 and folacin, is one of the $\mathrm{B}$ vitamins. Folate deficiency may lead to congenital malformations, anemia, spontaneous abortions, preeclampsia, intrauterine growth restriction, and placental abruption. Clinicians commonly recommend that patients start or continue to take prenatal vitamins with folic acid supplementation in order to prevent neural tube defects and preeclampsia. A daily supplemental dose of $400 \mu \mathrm{g} /$ day of folic acid is recommended when planning pregnancy, starting from 2 months before to 3 months after conception [121]. Women at high risk for folate deficiency should receive supplemental folic acid at 4-5 mg/day [173-177]. Evidence demonstrates that low dietary folate is associated with high risk of miscarriage [178], but the risk of early or late miscarriage is not reduced with folic acid supplementation alone or with other combinations according to a metaanalysis, and there appears to be no difference between women receiving folic acid and those who do not in terms of congenital malformations and stillbirths [24].

Vitamin $C$ is an essential nutrient involved in the repair of tissue and the enzymatic production of certain neurotransmitters, and it is vital for both maternal and fetal health. Foods high in vitamin $\mathrm{C}$ include citrus fruits, tomatoes, and broccoli, and a balanced diet that is high in Fe and vitamin $\mathrm{C}$ is beneficial for a healthy pregnancy. However, vitamin $\mathrm{C}$ levels tend to be low in women who have a miscarriage, and supplementation of vitamin $\mathrm{C}$ may reduce the risk of miscarriage [179]. Further, vitamin $C$ supplements increase maternal progesterone levels, improve psychological disorders, and may be of benefit to prevent miscarriage and decrease the development of preeclampsia $[180,181]$. Nevertheless, there is insufficient data to support the role of vitamin $\mathrm{C}$ supplementation alone or combined with vitamin $\mathrm{E}$ in reducing total fetal loss, the risk of early or late miscarriage, the risk of stillbirth, or the risk of congenital malformations or adverse events [24]. Supplementation with vitamin C may be beneficial for threatened miscarriage, but overdosing should be avoided [157].

Vitamin D mainly regulates the amount of calcium and phosphate needed to keep bones, teeth, and muscles functioning properly. Vitamin $\mathrm{D}$ requirements are increased during pregnancy in order to adapt to the heightened physiological demands in the mother, including driving the formation of the fetal skeleton and maintaining an environment that is tolerant of paternal and fetal tissues and their associated alloantigens $[182,183]$. It is estimated that $20 \%-60 \%$ of pregnant women in the UK, $10 \%-40 \%$ in the USA, and $30 \%-50 \%$ in Australia, India, and Saudi Arabia have a vitamin D deficiency [184-188]. Vitamin D deficiency may increase the risk of threatened miscarriage, and it is also related to adverse outcomes during pregnancy. Several observational studies demonstrated that serum 25-hydroxyvitamin $\mathrm{D}(25(\mathrm{OH}) \mathrm{D})$ levels in women with threatened miscarriage tend to be lower than those in women with normal pregnancy, suggesting that low serum levels of vitamin $\mathrm{D}$ can be considered a risk factor for threatened miscarriage [189-191]. Global reports suggest that 40\%-98\% of pregnant women have 25(OH)D levels below $50 \mathrm{nmol} / \mathrm{L}$ and 15\%-84\% have levels below $25 \mathrm{nmol} / \mathrm{L}$ [192, 193]. Importantly, vitamin D-binding protein (VDBP) levels are often low in maternal serum, and such deficiencies are associated with miscarriage and adverse pregnancy outcomes including preeclampsia, preterm birth, and fetal growth restriction [194]. VDBP is expressed at low levels in the placenta and decidua in spontaneous miscarriages, and it might serve as a potential biomarker for miscarriages and has implications in the pathophysiology of spontaneous miscarriage [195]. Supplementation with vitamin D seems to be beneficial for threatened miscarriage [157], and a randomized double-blinded study showed that supplementation with vitamin D3 (400 IU/day) led to a decreased incidence of miscarriage and serum IL-23 levels in women with unexplained recurrent spontaneous abortion [196]. Vitamin D supplementation can reduce inflammation and is useful as immunotherapy to prevent miscarriage by downregulating IL-2, IFN-gamma, and TNF-alpha gene transcription [197, 198]. The daily upper safe limit for vitamin D has been set at $4000 \mathrm{IU}$, and the recommended dose of vitamin D is 1000-2000 IU, which can be supplemented daily in the second and third trimesters without fear of toxicity or teratogenicity. However, no safety data are available for this dose during the first trimester [199].

Vitamin $E$ is important for the proper functioning of many organs, and it is an antioxidant that helps to inhibit processes that damage cells. Low vitamin E levels may be linked to greater miscarriage risk [200, 201]. Vitamin E in combination with aspirin is effective in improving uterine artery blood flow in women with recurrent miscarriage and thus prevents pregnancy loss and other complications [158]. A Hungarian case-control study reported that vitamin $\mathrm{E}$ supplements at a dose $450 \mathrm{mg}$ daily are frequently used for the prevention of threatened miscarriage and lead to a nearly one-third reduction in preterm births in pregnant women, although internationally this method is no longer recommended [202]. The use of vitamin E, although generally considered "healthy," may be harmful to the pregnancy outcome by disrupting the physiological oxidative gestational state and is consequently not recommended to prevent preeclampsia [157]. In a placebo-controlled, doubleblind trial, women diagnosed with chronic hypertension or who had a prior history of preeclampsia who received daily doses of vitamin E (400 IU) and vitamin C (1000 mg) had an increased risk of premature rupture of membranes [203], while another study did not confirm the teratogenic effects of the relatively high-dose vitamin $\mathrm{E}$ intake in pregnant women [204]. Further studies are needed on the effect and safety of vitamin $\mathrm{E}$ for threatened miscarriage and during early pregnancy. 
Multivitamin supplements are widely used by pregnant women. Multivitamins together with iron and folic acid are commonly recommended to improve birth outcomes and reduce the risk of miscarriage, and Cochrane reviews have shown that a multivitamin plus iron and folic acid can decrease the risk of stillbirth but with no significant effect on the overall risk of fetal loss or miscarriage compared with placebo, folic acid, or vitamin A alone [24, 122]. In all other analyses of the effect of multivitamin supplementation on total fetal loss or early or late miscarriage, there were no differences between groups, including multivitamins versus control, multivitamins with vitamin $\mathrm{E}$ versus multivitamins without vitamin $\mathrm{E}$ or control, and multivitamins with $\mathrm{Fe}$ and folic acid versus $\mathrm{Fe}$ and folic acid alone. Only a few trials found that multivitamins with or without vitamin A can reduce total fetal loss; however, these findings should be interpreted with caution due to small sample sizes [24]. In addition, studies have suggested that the use of multivitamin supplements within the range of the dietary reference intakes does not result in excess intake and does not increase mortality, and only minor adverse effects such as gastrointestinal symptoms have been reported with multivitamin supplements [125, 205].

4.3. Other Antioxidants. Oxidative stress has been recognized as one of the main mediators of female infertility by causing various reproductive pathologies such as polycystic ovary syndrome (PCOS), preeclampsia, miscarriage, and unexplained infertility [123]. When oxidative stress develops too early in pregnancy, it can impair placental development and/or enhance syncytiotrophoblastic degeneration, culminating in pregnancy loss [206]. Nowadays, concerned women prefer dietary supplements with antioxidant properties over synthetic drugs as a natural way to lessen the oxidative stress and enhance their fertility and to prevent early pregnancy loss, with the idea of replacing depleted antioxidant stores to combat an overwhelmingly oxidative environment. However, a meta-analysis of relevant studies found no supporting evidence for any beneficial effects of antioxidant supplementation [207]. Thus, caution must be still used in the application of antioxidants during pregnancy. The most commonly used antioxidants for pregnant women are vitamins, alpha-lipoic acid (ALA), and N-acetylcysteine (NAC), and future randomized controlled clinical trials in humans based on current animal or in vitro studies are necessary to elucidate the precise mechanisms through which oxidative stress affects female reproduction.

$A L A$ is a natural antioxidant synthesized by plants and animals [208], and it has been shown to prevent miscarriage by reducing the levels of proinflammatory cytokines, such as TNF-alpha, IL-1 beta, IL-6, IL-8, IL-17, and INF-gamma, and by inducing anti-inflammatory IL-10 release. In addition, ALA can induce vascular endothelial growth factor to stimulate tissue epithelialization, collagen deposition, and smooth muscle actin fibrogenesis in order to resolve subchorionic hematoma in threatened miscarriage [209-212]. ALA is able to reduce ROS levels and increase the total antioxidant capacity in cultured preantral mouse follicles
[213]. In randomized controlled trials, ALA supplementation alone at a dose of $10 \mathrm{mg}$ or $600 \mathrm{mg}$ or combined with vaginal progesterone could prevent pregnancy loss; reduce the symptoms of vaginal bleeding, abdominal pain, and uterine contractions; and promote chorionic hematoma reabsorption in pregnant women with threatened miscarriage compared to pregnant women without intervention or pregnant women treated with progesterone alone $[214,215]$. However, there was no statistical difference in the improvement of symptoms of threatened miscarriage, and the sample size was small. In order to further confirm the role of ALA in improving threatened miscarriage in pregnant women and to verify its safety, well-designed clinical studies with larger sample sizes should be conducted $[208,209,216]$.

NAC is a commonly used mucolytic drug, and it not only increases cellular antioxidant levels, but also improves insulin receptor activity in human erythrocytes and regulates the secretion of insulin in response to glucose uptake [217]. The supplement can prevent endothelial damage and biological effects caused by oxidants in non-insulin-dependent adult diabetics by preventing ischemia, inhibiting phospholipid metabolism, and promoting inflammatory cytokine release and protease activity [218]. NAC might have potential benefit for threatened miscarriage, and some studies have shown that NAC significantly improves the pregnancy rate and ovulation rate compared with placebo [218, 219]. However, some studies have shown that there is no significant difference in miscarriage rate between NAC supplementation and placebo in PCOS patients [218]. In addition, in a randomized controlled trial of PCOS patients with clomiphene citrate resistance, the live birth rate was higher after treatment with a combination of metformin and clomiphene citrate compared to treatment with NAC [220]. NAC was suggested to reduce fetal loss in a PCOS rat model; however, it was shown that supplementation with NAC could induce miscarriage in control pregnant rats [221]. Considering the poor quality of existing studies and the lack of studies assessing miscarriage rates, well-designed randomized controlled trials for threatened miscarriage are needed.

Omega-3 is a fatty acid that may have potential for preventing miscarriage. In a prospective study, omega-3 combined with aspirin improved the uterine artery blood flow velocity in women with recurrent miscarriage [222]. However, the literature on the relationship between human serum omega- 3 concentration and reproduction is limited specifically to infertile people, and whether it can prevent miscarriage is still controversial $[223,224]$. Therefore, more research is needed to clarify the role of omega- 3 and how it can be used more effectively.

At present, there are few studies focusing on the role of nutritional supplements as treatments for threatened miscarriage. Overall, current studies indicate that deficiencies in several trace elements ( $\mathrm{Zn}, \mathrm{Cu}, \mathrm{Fe}, \mathrm{Mg}$, and $\mathrm{Mn}$ ) and vitamins (A, B, C, D, E, and folate) are associated with high risk of pregnancy loss. In particular, low levels of $\mathrm{Zn}, \mathrm{Cu}, \mathrm{Fe}$, and vitamin $\mathrm{D}$ may result in threatened miscarriage. Thus, proper supplementation with $\mathrm{Zn}, \mathrm{Cu}, \mathrm{Fe}$, vitamin $\mathrm{D}$, vitamin $\mathrm{E}$, and ALA may have efficacy as treatments for threatened 
TABLE 4: The efficiency and safety of various CAM approaches in the treatment of threatened miscarriage.

\begin{tabular}{|c|c|c|}
\hline CAM therapies & Efficiency & Safety and risk \\
\hline CHM & $\begin{array}{c}\text { CHM may improve endometrial function, inhibit uterine } \\
\text { contraction, regulate immune response, and promote the } \\
\text { development of the embryo. It is considered beneficial for } \\
\text { preventing and treating miscarriage with fewer adverse } \\
\text { events compared to current conventional western medicine } \\
\text { by being used alone or in combination with western } \\
\text { medicines. }\end{array}$ & $\begin{array}{l}\text { Most clinical trials have reported no adverse events or } \\
\text { significant risks, but systematic reviews have concluded } \\
\text { that no data are available to demonstrate the safety of } \\
\text { CHM for the mother or the infant with current } \\
\text { publications. In addition, animal studies indicate that } \\
\text { the frequently used CHMs have reproductive toxicity } \\
\text { for threatened miscarriage, including fetal resorption, } \\
\text { growth restriction, and congenital malformations. }\end{array}$ \\
\hline $\begin{array}{l}\text { Acupuncture and } \\
\text { moxibustion }\end{array}$ & $\begin{array}{l}\text { Acupuncture, acupoint sticking, auricular acupoint, } \\
\text { acupoint injection, acupoint catgut embedding, and } \\
\text { moxibustion are recognized as nonpharmacological } \\
\text { approaches, which can maintain pregnancy, and enhance } \\
\text { the effects with the CHM or western medicines. Most } \\
\text { studies show that acupuncture is safe and effective without } \\
\text { side effects. }\end{array}$ & $\begin{array}{l}\text { The safety of acupuncture in pregnancy is reasonably } \\
\text { well accepted, but there is still a lack of high-quality } \\
\text { scientific research to validate the safety and } \\
\text { effectiveness of acupuncture and moxibustion for } \\
\text { threatened miscarriage. There remains a debate } \\
\text { regarding the needling points that are historically } \\
\text { considered to be forbidden during pregnancy. }\end{array}$ \\
\hline $\begin{array}{l}\text { Nutritional } \\
\text { supplements }\end{array}$ & $\begin{array}{l}\text { Nutritional supplements are used as an auxiliary way of } \\
\text { supplying amino acids, trace elements, vitamins, and } \\
\text { minerals for maintaining maternal health and fetal } \\
\text { development. Proper supplementation with } \mathrm{Zn}, \mathrm{Cu}, \mathrm{Fe} \text {, } \\
\text { vitamin } \mathrm{D} \text {, vitamin } \mathrm{E} \text {, and ALA may have efficacy as } \\
\text { treatments for threatened miscarriage and to prevent } \\
\text { further complications and is convenient to supplement. }\end{array}$ & $\begin{array}{l}\text { Most of studies are of low quality and controversial } \\
\text { because of their small sample sizes, poor and } \\
\text { inconsistent reporting of methods, excessive } \\
\text { confounding factors, and lack of reporting of clinically } \\
\text { relevant outcomes such as live birth and adverse events. }\end{array}$ \\
\hline $\begin{array}{l}\text { Psychological } \\
\text { interventions }\end{array}$ & $\begin{array}{c}\text { Psychological interventions may help women to regulate } \\
\text { their emotions and promote psychosocial well-being during } \\
\text { the current pregnancy or after a miscarriage, and this can be } \\
\text { beneficial for the offspring. }\end{array}$ & $\begin{array}{l}\text { There is little evidence to suggest that psychological } \\
\text { interventions are beneficial in improving psychological } \\
\text { morbidity to prevent threatened miscarriage. }\end{array}$ \\
\hline
\end{tabular}

CAM: complementary and alternative medicine; CHM: Chinese herbal medicine; Zn: zinc; Cu: copper; Fe: iron; ALA: alpha-lipoic acid.

miscarriage and in preventing further complications. Although many studies have sought to clarify the positive effects of nutritional supplements such as trace elements, vitamins, and antioxidants on threatened miscarriage, most of them are of low quality and controversial because of their small sample sizes, poor and inconsistent reporting of methods, excessive confounding factors, and lack of reporting of clinically relevant outcomes such as live birth and adverse events. Therefore, it is necessary to carefully design more high-quality studies to clarify the specific effects of nutritional supplements on threatened miscarriage, as well as their safety and tolerance, so as to better provide care for pregnant women. Moreover, there is currently insufficient evidence to determine the effects of different combinations of nutritional supplements on threatened miscarriage.

\section{Psychological Interventions and Other CAM Therapies in Threatened Miscarriage}

Of all clinically recognized pregnancies, a quarter to a third experience a threatened miscarriage. Miscarriage is often a physically and psychologically traumatic event for these women and their families, and women may suffer psychological morbidities such as stress, anxiety, depression, and grief as a reaction to miscarriage [225]. The psychological distress can last between one and three years after a miscarriage and may affect the woman's quality of life and subsequent attempts to become pregnant [226]. Additionally, psychological distress can impact the fetus and result in preterm birth or low birth weight, and it can result in longterm consequences for the child such as deficits in cognitive functioning and an increase in negative behavior [227, 228]. Despite the potential of follow-up care or emotional support for negative outcomes, more than half of women do not receive psychological interventions at the time of miscarriage. [229], thus there is a need to find ways to mitigate psychological distress in order to prevent miscarriage.

CAM approaches may help women to regulate their emotions during the current pregnancy or after a miscarriage, which can be beneficial for the offspring, and it is reported that approximately $40 \%$ of women with a history of miscarriage have used a CAM approach [8]. Psychological interventions or supportive care can help women who have had a miscarriage to overcome their grief and can promote psychosocial well-being, and in a cross-sectional survey distributed to pregnant women residing in the USA, the most frequently reported complementary approaches used by pregnant women with a history of miscarriage were prayer $(22.3 \%)$, yoga (15\%), massage $(14.5 \%)$, chiropractic treatment $(13 \%)$, and meditation $(11.4 \%)$ [8, 230]. Similarly, massage, yoga, and relaxation are the most commonly reported complementary approaches used by pregnant women in Australia [231], while homeopathy, acupuncture, and phytotherapy are the most frequently used during pregnancy in Germany [232]. However, there is little evidence to suggest that CAM therapies are beneficial in improving psychological morbidity to prevent threatened miscarriage, although complementary approaches may upregulate dopamine levels and decrease stress signaling hormones that in 
turn control mood $[233,234]$. Future research in this area is needed to establish the effectiveness of complementary approaches in pregnant women and to provide evidence for strategies that healthcare providers can use when treating their patients.

\section{Summary}

We have reviewed the primary literature focusing on the research into the application of CAM in women with threatened miscarriage, with an emphasis on the efficacy and safety of the treatments. In general, CHM, acupuncture and moxibustion, nutritional supplements, and psychological interventions are the most commonly used to prevent pregnancy loss or complications, and they have proven beneficial for the treatment of threatened miscarriage. We briefly summarize the efficacy and safety of various CAM approaches in the treatment of threatened miscarriage (Table 4). Commonly, the CAM therapies are combined with luteal support medications such as progesterone and/or hCG for threatened miscarriage. It is suggested that pregnancy loss had a deficiency of progesterone and hCG, and low levels of maternal serum progesterone or hCG in early pregnancy may be adverse prognostic factors for threatened miscarriage $[12,13,235]$. Hence, supplementation with progesterone or hCG may prevent miscarriage and maintain pregnancy; however, the evidence remains conflicting. Although the meta-analysis indicated that progesterone may reduce the rate of spontaneous miscarriage but has little effect in the preterm birth, and hCG may have no significant effect in women with threatened miscarriage, the evidence is insufficient and of poor quality [12, 13, 236]. Currently, luteal support medications are still main management for threatened miscarriage in clinic. Moreover, majority of data show that the combination of CAM and luteal support medications may increase maternal circulation progesterone and hCG levels, which seems to be more beneficial than that medication alone for maintaining pregnancy and promoting psychosocial well-being in women with threatened miscarriage. However, whether the use of CAMs is main or adjuvant therapy is still unsure. Therefore, not only CAM approaches but also luteal support drugs need further research with goodquality evidence to determine the effects in threatened miscarriage.

However, issues of safety and risks with the use of CAM during early pregnancy require further studies. At present, there is little evidence to indicate whether CHM, acupuncture, and supplements are harmful for fetal development or whether they induce pregnancy loss, premature delivery, or stillbirth. It would be beneficial to conduct largescale, randomized clinical trials in the future to determine the efficacy and safety of CAM, to help substantiate its therapeutic effects, and to identify possible adverse events. As the Chinese idiom goes "take the essence and discard the dregs," we need further investigations to develop effective and safe CAM therapy regimes during pregnancy to support both the mother and her baby.

\section{Conflicts of Interest}

The authors declare that there are no conflicts of interest regarding the publication of this paper.

\section{Authors' Contributions}

Min $\mathrm{Hu}$ contributed to the original draft and takes responsibility for the integrity of the final manuscript. Lingjing $\mathrm{Lu}, \mathrm{Yu}$ Zhou, and Min $\mathrm{Hu}$ collected the references and drafted the manuscript. Juan Li and Hongxia Ma revised the manuscript. All authors have read and approved the final version of the manuscript.

\section{Acknowledgments}

This work was supported by the National Natural Science Fund of China (grant no. 82004399), the Guangdong Basic and Applied Basic Research Foundation (grant no. 2019A1515110265), and the Guangzhou Medical University High-Level University Construction Talents Fund (grant no. B185006010046).

\section{References}

[1] F. Blohm, B. Fridén, and I. Milsom, "A prospective longitudinal population-based study of clinical miscarriage in an urban Swedish population," BJOG: An International Journal of Obstetrics and Gynaecology, vol. 115, no. 2, pp. 176-183, 2008.

[2] G. E. Robinson, "Pregnancy loss," Best Practice \& Research Clinical Obstetrics \& Gynaecology, vol. 28, no. 1, pp. 169-178, 2014.

[3] C. Everett, "Incidence and outcome of bleeding before the 20th week of pregnancy: prospective study from general practice," BMJ, vol. 315, no. 7099, pp. 32-34, 1997.

[4] L. Saraswat, S. Bhattacharya, A. Maheshwari, and S. Bhattacharya, "Maternal and perinatal outcome in women with threatened miscarriage in the first trimester: a systematic review," BJOG: An International Journal of Obstetrics \& Gynaecology, vol. 117, no. 3, pp. 245-257, 2010.

[5] J. L. Weiss, F. D. Malone, J. Vidaver et al., "Threatened abortion: a risk factor for poor pregnancy outcome, a population-based screening study," American Journal of Obstetrics and Gynecology, vol. 190, no. 3, pp. 745-750, 2004.

[6] R. H. F. van Oppenraaij, E. Jauniaux, O. B. Christiansen, J. A. Horcajadas, R. G. Farquharson, and N. Exalto, "Predicting adverse obstetric outcome after early pregnancy events and complications: a review," Human Reproduction Update, vol. 15, no. 4, pp. 409-421, 2009.

[7] J. Johns, S. Muttukrishna, M. Lygnos, N. Groome, and E. Jauniaux, "Maternal serum hormone concentrations for prediction of adverse outcome in threatened miscarriage," Reproductive BioMedicine Online, vol. 15, no. 4, pp. 413-421, 2007.

[8] J. Huberty, J. Matthews, J. A. Leiferman, and C. Lee, "Use of complementary approaches in pregnant women with a history of miscarriage," Complementary Therapies in Medicine, vol. 36, pp. 1-5, 2018.

[9] D. Mayrhofer, M. Hager, K. Walch et al., "The prevalence and impact of polycystic ovary syndrome in recurrent miscarriage: a retrospective cohort study and meta-analysis," Journal of Clinical Medicine, vol. 9, no. 9, p. 2700, 2020. 
[10] L. Li, P. C. Leung, T. K. Chung et al., "Systematic review of Chinese medicine for miscarriage during early pregnancy," Evidence-Based Complementary and Alternative Medicine, vol. 2014, Article ID 753856, 16 pages, 2014.

[11] D. Betts, C. A. Smith, and D. G. Hannah, "Acupuncture as a therapeutic treatment option for threatened miscarriage," BMC Complementary Medicine and Therapies, vol. 12, p. 20, 2012.

[12] H. A. Wahabi, A. A. Fayed, S. A. Esmaeil, and K. H. Bahkali, "Progestogen for treating threatened miscarriage," The Cochrane Database of Systematic Reviews, vol. 8, Article ID CD005943, 2018.

[13] A. Sotiriadis, S. Papatheodorou, and G. Makrydimas, "Threatened miscarriage: evaluation and management," BMJ, vol. 329, no. 7458, pp. 152-155, 2004.

[14] J. Sarris, T.-B. Robins Wahlin, D. Goncalves, and G. Byrne, "Comparative use of complementary medicine, allied health, and manual therapies by middle-aged and older Australian women," Journal of Women \& Aging, vol. 22, no. 4, pp. 273-282, 2010.

[15] C. A. Lunny and S. N. Fraser, "The use of complementary and alternative medicines among a sample of Canadian menopausal-aged women," Journal of Midwifery \& Women's Health, vol. 55, no. 4, pp. 335-343, 2010.

[16] D. M. Eisenberg, R. B. Davis, S. L. Ettner et al., "Trends in alternative medicine use in the United States, 1990-1997: results of a follow-up national survey," JAMA, vol. 280, no. 18, pp. 1569-1575, 1998.

[17] T. Volqvartz, A. L. Vestergaard, S. K. Aagaard et al., "Use of alternative medicine, ginger and licorice among Danish pregnant women-a prospective cohort study," BMC Complementary Medicine and Therapies, vol. 19, no. 1, p. 5, 2019.

[18] G. S. Birdee, K. J. Kemper, R. Rothman, and P. Gardiner, "Use of complementary and alternative medicine during pregnancy and the postpartum period: an analysis of the national health interview survey," Journal of Women's Health, vol. 23, no. 10, pp. 824-829, 2014.

[19] A. R. Pallivalappila, D. Stewart, A. Shetty et al., "Complementary and alternative medicines use during pregnancy: a systematic review of pregnant women and healthcare professional views and experiences," Evidence-Based Complementary and Alternative Medicine, vol. 2013, Article ID 205639, 10 pages, 2013.

[20] J. Frawley, J. Adams, D. Sibbritt, A. Steel, A. Broom, and C. Gallois, "Prevalence and determinants of complementary and alternative medicine use during pregnancy: results from a nationally representative sample of Australian pregnant women," Australian and New Zealand Journal of Obstetrics and Gynaecology, vol. 53, no. 4, pp. 347-352, 2013.

[21] S. M. Illamola, O. U. Amaeze, L. V. Krepkova et al., "Use of herbal medicine by pregnant women: what physicians need to know," Frontiers in Pharmacology, vol. 10, p. 1483, 2019.

[22] M. El Hajj and L. Holst, "Herbal medicine use during pregnancy: a review of the literature with a special focus on sub-Saharan Africa," Frontiers in Pharmacology, vol. 11, p. 866,2020 .

[23] C. Savona-Ventura and T. Mahmood, "EBCOG position statement about the use of herbal medication during pregnancy," European Journal of Obstetrics \& Gynecology and Reproductive Biology, vol. 244, pp. 38-39, 2020.

[24] O. O. Balogun, K. da Silva Lopes, E. Ota et al., "Vitamin supplementation for preventing miscarriage," Cochrane
Database of Systematic Reviews, vol. 2016, Article ID CD004073, 2016.

[25] L. J. John and N. Shantakumari, "Herbal medicines use during pregnancy: a review from the Middle East," Oman Medical Journal, vol. 30, no. 4, pp. 229-236, 2015.

[26] D. A. Kennedy, A. Lupattelli, G. Koren et al., "Safety classification of herbal medicines used in pregnancy in a multinational study," BMC Complementary Medicine and Therapies, vol. 16, p. 102, 2016

[27] L. Li, L. X. Dou, J. P. Neilson, P. C. Leung, and C. C. Wang, "Adverse outcomes of Chinese medicines used for threatened miscarriage: a systematic review and meta-analysis," Human Reproduction Update, vol. 18, no. 5, pp. 504-524, 2012.

[28] L. Li, L. Dou, P. C. Leung et al., "Chinese herbal medicines for threatened miscarriage," Cochrane Database of Systematic Reviews, vol. 5, Article ID CD008510, 2012.

[29] L. Li, L. Dou, P. C. Leung et al., "Chinese herbal medicines for unexplained recurrent miscarriage," Cochrane Database of Systematic Reviews, vol. 1, Article ID CD010568, 2016.

[30] H. Ping, "Clinical study on combination of traditional Chinese medicine and western medicine in treating early threatened abortion," China Journal of Chinese Medicine, vol. 9, pp. 1199-1201, 2012.

[31] G. Y. Yang, H. Luo, X. Liao et al., "Chinese herbal medicine for the treatment of recurrent miscarriage: a systematic review of randomized clinical trials," BMC Complementary Medicine and Therapies, vol. 13, p. 320, 2013.

[32] China Medical Science Press, Pharmacopoeia of the People's Republic of China, China Medical Science Press, Beijing, China, 2015.

[33] F. Gao, H. Wu, J. Gao et al., "Shoutai pill for threatened miscarriage: meta analysis of randomized controlled trials," Chinese Archives of Traditional Chinese Medicine, vol. 34, no. 11, pp. 2637-2642, 2016.

[34] H. Niu and H. Cheng, "Clinical observation of modified Shoutai pills in treating kidney deficiency type threatened abortion," Clinical Journal of Traditional Chinese Medicine, vol. 30, no. 2, pp. 324-326, 2018.

[35] J. Zhu, Y. Zhang, X. Li et al., "Clinical observation on the effect of Shoutai pills plus decoction combined with progesterone on the treatment of early threatened abortion patients," Yunnan Journal of Traditional Chinese Medicine and Materia Medica, vol. 37, no. 12, pp. 26-28, 2016.

[36] L. Sun, M. Wei, K. Liang et al., "The treatment clinical results of abortion early about Shoutaisijunzi decoction and progesterone," Pharmacology and Clinics of Chinese Materia Medica, vol. 32, no. 2, pp. 222-224, 2016.

[37] Y. Ye, "Clinical observation of Shoutai pills plus progesterone in the treatment of early threatened abortion," Journal of Practical Traditional Chinese Medicine, vol. 33, no. 3, pp. 257-258, 2017.

[38] F. Han, J. Li, M. Tian et al., "Clinical observation on 60 cases of threatened abortion treated by Zishen Yutai pills combined with progesterone injection," World Chinese Medicine, vol. 13, no. 4, pp. 826-829, 2018.

[39] J. N. Lin, X. L. Liao, and H. J. Han, “Observation on efficacy of Zishen Yutai pills combined with dydrogesterone tablets in treatment of early threatened abortion," Evaluation and Analysis of Drug-Use in Hospitals of China, vol. 17, no. 4, pp. 459-461, 2017.

[40] X. Liu and Z. Liu, "Clinical observation of Zishen Yutai pills combined with dydrogesterone in the treatment of threatened abortion with spleen and kidney deficiency," Journal of 
Practical Traditional Chinese Medicine, vol. 35, no. 8, pp. 1003-1004, 2019.

[41] Z. Tang, X. Zhang, and R. Mu, "Clinical observation on treating threatened abortion with Zishen Yutai pills plus progesterone capsule," Clinical Journal of Chinese Medicine, vol. 7, no. 4, pp. 55-57, 2015.

[42] S. Wang, "Clinical study of Zishen Yutai pills in the treatment of threatened abortion and subchorionic hemorrhage in the first trimester," Hebei Medicine, vol. 22, no. 5, pp. 810-812, 2016.

[43] Y. F. Liu, "Clinical observation of Zishen Yutai pills combined with allylestrenol in treatment of threatened abortion," Drugs \& Clinic, vol. 33, no. 6, pp. 1444-1447, 2018.

[44] Y. Ouyang and L. Jia, "Effect on blood rheology and uterine placenta perfusion of Zishen Yutai pills combined with progesterone tablet in the treatment of patients with early threatened abortion," Medical Innovation of China, vol. 13, no. 8, pp. 19-22, 2016.

[45] C. Tian and B. Chen, "Effects of Gushen Antai pills combined with progestin on serum $\beta$-HCG, P, E2 and CA125 in patients with threatened abortion," China Journal of Chinese Materia Medica, vol. 41, no. 2, pp. 321-325, 2016.

[46] J. W. Zhang and L. Qian, "Observation on the efficacy of Gushen Antai pills combined with dydrogesterone in the treatment of early threatened abortion," Electronic Journal of Practical Gynecologic Endocrinology, vol. 3, no. 3, pp. 51-52, 2016.

[47] M. Shen, N. Li, Z. Lu et al., "Effect and mechanism of Gushen Antai pills combined with progesterone in threatened abortion patients," Northwest Pharmaceutical Journal, vol. 35, no. 1, pp. 109-112, 2020.

[48] W. Wang and J. Dang, "60 cases of early threatened abortion treated with Gushen Antai pills combined with progesterone soft capsule," Shaanxi Journal of Traditional Chinese Medicine, vol. 37, no. 11, pp. 1451-1452, 2016.

[49] Z. Li, "Treatment of 70 cases of threatened miscarriage with modified Andian Ertian decoction and progesterone injection," Henan Traditional Chinese Medicine, vol. 34, no. 7, pp. 1370-1371, 2014.

[50] Y. Wang, "Curative observation of using plus-minus Andian Ertian decoction to treat threatened abortion," Journal of Sichuan of Traditional Chinese Medicine, vol. 32, no. 9, pp. 107-109, 2014.

[51] L. Guo and J. Guo, "Observation on the efficacy of Baoyin decoction combined with progesterone in the treatment of threatened abortion due to insufficient corpus luteum function," Journal of Practical Traditional Chinese Medicine, vol. 33, no. 7, p. 780, 2017.

[52] X. Li, X. Hu, Q. Yang et al., "Effects of Baoyin decoction on serum P, $\beta$-HCG, CA125 in patients with early threatened abortion," World Chinese Medicine, vol. 13, no. 8, pp. 1887-1890, 2018.

[53] H. Lv and H. Wu, "Observation on the therapeutic effect of Baotai decoction in treating 60 cases of threatened miscarriage due to luteal deficiency," Zhejiang Journal of Traditional Chinese Medicine, vol. 54, no. 4, p. 270, 2019.

[54] W. Li, D. Li, Y. Lu et al., "Observation on curative effect of Bushen Antai decoction in treating early threatened abortion," Clinical Journal of Traditional Chinese Medicine, vol. 3, pp. 232-233, 2004.

[55] S. Lv, "Treating 77 cases of threatened abortion with Bushen Antai decoction and progesterone," Henan Traditional Chinese Medicine, vol. 32, no. 10, pp. 1361-1362, 2012.
[56] X.-H. Zhong, Z.-X. Zhou, T.-S. Li, E.-Q. Wang, W.-Y. Shi, and S.-M. Chu, "Anti-abortive effect of radix Scutellariae and rhizoma Atractylodis in mice," The American Journal of Chinese Medicine, vol. 30, no. 1, pp. 109-117, 2002.

[57] X. Y. Tian, L. M. Cheung, K. Leung et al., "The effects of Scutellaria baicalensis extract on embryonic development in mice," Birth Defects Research Part B: Developmental and Reproductive Toxicology, vol. 86, no. 2, pp. 79-84, 2009.

[58] A.-T. Ma, X.-H. Zhong, Z.-M. Liu et al., "Protective effects of baicalin against bromocriptine induced abortion in mice," The American Journal of Chinese Medicine, vol. 37, no. 1, pp. 85-95, 2009.

[59] L. Deng, D. Song, J. Guo et al., "Study on ingredients of Scutellaria radix extract penetrable through placental barrier of pregnant rat," Zhongguo Zhong Yao Za Zhi, vol. 37, no. 3, pp. 327-330, 2012.

[60] J. Zhang, G. Chu, X. Liu et al., "Effects of Danggui san on isolated smooth muscle of uterus and uterine smooth muscle strips stimulated by oxytocin in rats," Chinese Journal of Experimental Traditional Medical Formulae, vol. 17, no. 7, pp. 201-203, 2011.

[61] S. Yang, X. Xu, H. Xu et al., "Purification, characterization and biological effect of reversing the kidney-yang deficiency of polysaccharides from semen cuscutae," Carbohydrate Polymers, vol. 175, pp. 249-256, 2017.

[62] H. X. Ma, Z. L. You, and X. Y. Wang, "Effect of total flavones from Cuscuta chinensis on expression of Fas/FasL, PCNA and HB-EGF in SD rats model with bromocriptine-induced abortion," Zhong Yao Cai, vol. 31, no. 11, pp. 1706-1709, 2008.

[63] F. Gao, C. Zhou, W. Qiu et al., "Total flavonoids from semen Cuscutae target MMP9 and promote invasion of EVT cells via Notch/AKT/MAPK signaling pathways," Scientific Reports, vol. 8, no. 1, p. 17342, 2018.

[64] X. Li, "Study on genotoxicity of Herba Taxilli," Chinese Journal of Birth Health \& Heredity, vol. 16, no. 12, pp. 46-48, 2018.

[65] L. Li, J. Guan, J. Feng et al., "Study on the anti-rheumatism effective substances and meridian tropism of the total flavonoids of Taxilluschinensis (DC.) based on "syndromeefficacy-analysis of biological samples"” China Journal of Traditional Chinese Medicine and Pharmacy, vol. 33, no. 5, pp. 2203-2208, 2018.

[66] Z. Li and W. Li, "Effect of composite extract of donkey-hide gelatin on anemia animal models," Acta Chinese Medicine, no. 2, pp. 33-34, 2004.

[67] W. Huang, Q. Zeng, Z. Pan et al., "Study on the main pharmacodynamics and acute toxicity of eucommia leaves electuary," Guizhou Medical Journal, vol. 6, pp. 325-326, 2000.

[68] G. Lv, Y. Li, W. Li et al., "Effects of cortex Eucommiae extracts on estradiol-inducecd kidney-yang deficiency mice," Zhejiang Journal of Integrated Traditional Chinese and Western Medicine, vol. 19, no. 1, pp. 1-4, 2009.

[69] X. Gong, H. Ji, Q. Wang et al., "Anti-spasticity and antiabortion effects of total alkaloids of Dipsacus asper on uterus of pregnant rats," Journal of China Pharmaceutical University, vol. 29, no. 6, pp. 459-461, 1998.

[70] J.-S. Han, B.-S. Lee, S.-R. Han et al., "A subchronic toxicity study of radix Dipsaci water extract by oral administration in F344 rats," Regulatory Toxicology and Pharmacology, vol. 81, pp. 136-145, 2016.

[71] L. Y. Tang, L. Li, A. Borchert, C. B. S. Lau, P. C. Leung, and C. C. Wang, "Molecular studies of the congenital 
malformation induced by largehead Atractylodes rhizome, the most commonly used Chinese medicine for threatened miscarriage," Molecular Human Reproduction, vol. 18, no. 12, pp. 585-592, 2012.

[72] R. Wang, Study on the Molecular Mechanism of the Components of Milkvitch-Red Sage Compound Elevating Placenta Blood Supply, Hunan University of Chinese Medicine, Changsha, China, 2003.

[73] X. J. Zhang, C. C. Zhu, L. Hu et al., "Pharmacological action of polysaccharides from radix Codonopsis on immune function and hematopoiesis in mice," Traditional Chinese Drug Research and Clinical Pharmacology, vol. 3, pp. 174176, 2003.

[74] W. Xu, L. Xu, B. Deng et al., "The potential impact of radix Paeoniae Alba in embryonic development of mice," Phytotherapy Research, vol. 31, no. 9, pp. 1376-1383, 2017.

[75] C. C. Wang, L. Li, L. Y. Tang, and P. C. Leung, "Safety evaluation of commonly used Chinese herbal medicines during pregnancy in mice," Human Reproduction, vol. 27, no. 8, pp. 2448-2456, 2012.

[76] L. Li, L. Y. Tang, G. C. W. Man et al., "Potential reproductive toxicity of largehead Atractylodes rhizome, the most commonly used Chinese medicine for threatened miscarriage," Human Reproduction, vol. 26, no. 12, pp. 3280-3288, 2011.

[77] T. E. Strandberg, A. L. Jarvenpaa, H. Vanhanen et al., "Birth outcome in relation to licorice consumption during pregnancy," American Journal of Epidemiology, vol. 153, no. 11, pp. 1085-1088, 2001.

[78] H. F. Li, Q. H. Shen, X. Q. Li et al., "The efficacy of traditional Chinese medicine Shoutai pill combined with western medicine in the first trimester of pregnancy in women with unexplained recurrent spontaneous abortion: a systematic review and meta-analysis," BioMed Research International, vol. 2020, Article ID 7495161, 13 pages, 2020.

[79] L. Zhao, Y. Xu, X. Duo, X. Tian, and P. Wang, "A probe into effect of Shoutai pill on endometrial receptivity," Experimental and Therapeutic Medicine, vol. 15, no. 4, pp. 37653768, 2018.

[80] Y. Li, X. Y. Liu, J. L. Wang et al., "Effects of Shoutai pill containing serum on bioactivity behavior of trophoblast cells of spontaneous abortion patients," Zhongguo Zhong Xi Yi Jie He Za Zhi, vol. 36, no. 5, pp. 586-591, 2016.

[81] J. Zhang, L. Chen, C.-h. Zheng, J. Wang, D. Xie, and Y.-x. Zhou, "Effect of Shoutai pills on Th1/Th2 cytokines in serum and endometrium of rats with stimulated ovulation," Current Medical Science, vol. 39, no. 2, pp. 285-290, 2019.

[82] M. Jin, J. Chuan, Y. Shen et al., "Effects of Shoutai pills on immune function and oxidative stress in pregnant rats with di(2-ethylhexyl) phthalate exposure," Nan Fang Yi Ke Da Xue Xue Bao, vol. 40, no. 6, pp. 850-855, 2020.

[83] Q. Gu, X. Han, and Y. Geng, “TCM-Bushenantai treatment of rat model with threatened abortion," Jilin Journal of Traditional Chinese Medicine, vol. 36, no. 3, pp. 298-300, 2016.

[84] A. Wiebrecht, W. Gaus, S. Becker, J. Hummelsberger, and K. Kuhlmann, "Safety aspects of Chinese herbal medicine in pregnancy-re-evaluation of experimental data of two animal studies and the clinical experience," Complementary Therapies in Medicine, vol. 22, no. 5, pp. 954-964, 2014.

[85] Y. P. Zhu, T. B. Zhang, X. Y. Wan et al., "Teratogenicity study of astragaloside in SD rats," Chinese Traditional Patent Medicine, vol. 32, no. 10, pp. 1783-1785, 2010.
[86] Y. P. Hu, X. Wang, J. Song et al., "Genotoxicity study of ortex eucommiae decoction," West China Journal of Pharmaceutical Sciences, vol. 24, no. 5, pp. 490-493, 2009.

[87] L. Zhou, J. Zhou, J. Jiang et al., "Reproductive toxicity of ZishenYutai pill in rats: perinatal and postnatal development study," Regulatory Toxicology and Pharmacology, vol. 81, pp. 120-127, 2016.

[88] H. Williams, L. Sweet, and K. Graham, "Acupuncture during pregnancy and the perinatal period: women's attitudes, beliefs and practices," Women and Birth, vol. 33, no. 3, pp. e286-e294, 2020.

[89] D. Betts, M. Armour, and N. Robinson, "U.K. support network for maternity acupuncture: survey of acupuncturists on the acupuncture (for conception to) childbirth team," Medical Acupuncture, vol. 31, no. 5, pp. 274-280, 2019.

[90] C. A. Smith, J. M. Ussher, J. Perz, B. Carmady, and S. de Lacey, "The effect of acupuncture on psychosocial outcomes for women experiencing infertility: a pilot randomized controlled trial," The Journal of Alternative and Complementary Medicine, vol. 17, no. 10, pp. 923-930, 2011.

[91] P. C. Magarelli, D. K. Cridennda, and M. Cohen, "Changes in serum cortisol and prolactin associated with acupuncture during controlled ovarian hyperstimulation in women undergoing in vitro fertilization-embryo transfer treatment," Fertility and Sterility, vol. 92, no. 6, pp. 1870-1879, 2009.

[92] D. Betts, C. A. Smith, and H. G. Dahlen, "Does acupuncture have a role in the treatment of threatened miscarriage? Findings from a feasibility randomised trial and semistructured participant interviews," BMC Pregnancy Childbirth, vol. 16, no. 1, p. 298, 2016.

[93] D. Betts, C. A. Smith, and H. G. Dahlen, “"Well I'm safe because..."-acupuncturists managing conflicting treatment recommendations when treating threatened miscarriage: a mixed-methods study," The Journal of Alternative and Complementary Medicine, vol. 20, no. 11, pp. 838-845, 2014.

[94] R. C. Niemtzow, D. Betts, S. Budd, C. Citkovitz, Z. Kocher, and C. Mummery, "Acupuncture during pregnancy: an expert discussion," Medical Acupuncture, vol. 31, no. 5, pp. 251-258, 2019.

[95] D. J. Carr, "The safety of obstetric acupuncture: forbidden points revisited," Acupuncture in Medicine, vol. 33, no. 5, pp. 413-419, 2015.

[96] J. Park, Y. Sohn, A. R. White et al., "The safety of acupuncture during pregnancy: a systematic review," Acupuncture in Medicine, vol. 32, no. 3, pp. 257-266, 2014.

[97] H. Y. Moon, M. r. Kim, D. S. Hwang et al., "Safety of acupuncture during pregnancy: a retrospective cohort study in Korea," BJOG: An International Journal of Obstetrics \& Gynaecology, vol. 127, no. 1, pp. 79-86, 2020.

[98] L. Mårtensson, L. J. Kvist, and E. Hermansson, "A national survey of how acupuncture is currently used in midwifery care at Swedish maternity units," Midwifery, vol. 27, no. 1, pp. 87-92, 2011.

[99] K. Münstedt, J. Thienel, I. Hrogovic, A. Hackethal, M. Kalder, and B. Misselwitz, "Use of acupuncture and other CAM methods in obstetrics: an analysis of 409,413 deliveries from Hesse, Germany," The Journal of Alternative and Complementary Medicine, vol. 17, no. 5, pp. 421-426, 2011.

[100] J. Li and G. Xie, "Observation on 80 cases of incipient abortion treated with acupuncture via Lingguibafa and differential point selection," Shenzhen Journal of Integrated Traditional Chinese and Western Medicine, vol. 15, no. 2, pp. 106-107, 2005. 
[101] G. H. He, J. W. Ruan, and T. Xiang, "Effectiveness of acupoint sticking therapy in common chronic diseases, with disease differentiation principle," Annals of Palliative Medicine, vol. 3, no. 1, pp. 32-36, 2014.

[102] Y. Li, J. Yang, and H. Tan, "A systematic review and metaanalysis of the effectiveness and safety of acupuncture in the treatment of threatened miscarriage," Hunan Journal of Traditional Chinese Medicine, vol. 34, no. 7, pp. 164-169, 2018.

[103] Y. Huang, "Observation on 30 cases of threatened miscarrage treated by oral administration and external application of Dingtai Yin," Guiding Journal of Traditional Chinese Medicine and Pharmacy, vol. 16, no. 7, pp. 50-51, 2010.

[104] H. H. Huang, X. P. Yang, C. D. Yan et al., "Observation on the effect of Chinese medical sticking on acupoint Shenque on early threatened abortion," Guangxi Journal of Traditional Chinese Medicine, vol. 37, no. 1, pp. 27-28, 2014.

[105] G. Q. Shen and J. M. Chen, "Nursing intervention of Chinese medical sticking on acupoint Shenque combined with progestin on earlier threatened abortion due to kidney deficiency," Journal of Clinical Medicine in Practice, vol. 18, no. 22, pp. 85-88, 2014.

[106] G. Zhen, H. Zhang, W. Y. Li et al., "Nursing intervention of Shenque (CV 8) point sticking combined with progesterone injection on patients with kidney seficiency type early threatened abortion," Chinese Medicine Modern Distance Education of China, vol. 14, no. 11, pp. 124-125, 2016.

[107] X. F. Liu and X. D. Cui, "With Chinese medicine kidney and spleen side of acupoint sticking therapy clinical research threatened abortion," Clinical Journal of Traditional Chinese Medicine, vol. 28, no. 4, pp. 550-553, 2016.

[108] N. Soliman, B. L. Frank, H. Nakazawa, A. Averil, and R. Jodorkovsky, "Auricular acupuncture and auricular medicine acupuncture reflex systems of the ear, scalp, and hand," Physical Medicine and Rehabilitation Clinics of North America, vol. 10, no. 3, pp. 547-571, 1999.

[109] C. Ndubisi, A. Danvers, M. A. Gold, L. Morrow, and C. L. Westhoff, "Auricular acupuncture as an adjunct for pain management during first trimester abortion: a randomized, double-blinded, three arm trial," Contraception, vol. 99, no. 3, pp. 143-147, 2019.

[110] J. M. Wu, Y. Ning, J. Liao et al., "Effect on immune factors and serum CA125 in patients of threatened abortion in early pregnancy complicated with subchorionic hematoma treated with auricular acupuncture combined with dydrogesterone," Zhongguo Zhen Jiu, vol. 39, no. 10, pp. 1046-1050, 2019.

[111] J. Liao, T. Wang, W. Dong et al., "Acupoint injection for nonspecific chronic low back pain: a protocol of systematic review," Medicine (Baltimore), vol. 98, no. 29, Article ID e16478, 2019.

[112] Q. Wang, J. X. Xiong, and W. Y. Pan, "Observation on treatment of chronic pelvic inflammatory with point injection combined with ultra-laser radiation," Zhongguo Zhen Jiu, vol. 26, no. 9, pp. 614-616, 2006.

[113] Y. Zhao, Y. P. Wang, Q. L. Ding et al., "Effect of acupoint injection of human chorionic gonadotropins on sex hormones in patients with threatened abortion," Lishizhen Medicine and Materia Medica Research, vol. 19, no. 11, pp. 2795-2796, 2008.

[114] Y. Zhao, "Treatment of Zusanli acupoint injection of human chorionic gonadotropins on 36 cases of threatened abortion," Shaanxi Journal of Traditional Chinese Medicine, vol. 29, no. 7, pp. 875-877, 2008.
[115] Q. L. Ding, Y. Zhao, H. Y. Wu et al., "The effect of fluid acupuncture therapy on the level of sex serum hormone of threatened abortion patients," Hebei Journal of Traditional Chinese Medicine, vol. 30, no. 11, pp. 1187-1188, 2008.

[116] J. Sheng, X. Jin, J. Zhu et al., "The effectiveness of acupoint catgut embedding therapy for abdominal obesity: a systematic review and meta-analysis," Evidence-Based Complementary \& Alternative Medicine, vol. 2019, Article ID 9714313, 12 pages, 2019.

[117] J. M. Wu, Y. Chen, Y. Ning et al., “Adjuvant therapy of acupoint catgut embedding for threatened abortion after in vitro fertilization-embryo transfer: a randomized controlled trial," Zhongguo Zhen Jiu, vol. 39, no. 7, pp. 689-693, 2019.

[118] Y. Guo, "Clinical study of moxibustion combined with dydrogesterone in the treatment of early threatened miscarriage," Chinese Journal of Birth Health and Heredity, vol. 24, no. 4, pp. 88-90, 2016.

[119] Y. Zhang, "Explore the clinical effect of moxibustion combined with dydrogesterone in the treatment of early threatened miscarriage," Chinese Journal of Modern Drug Application, vol. 13, no. 21, pp. 209-210, 2019.

[120] M. Miranda-Garcia, C. Domingo Gómez, C. Molinet-Coll et al., "Effectiveness and safety of acupuncture and moxibustion in pregnant women with noncephalic presentation: an overview of systematic reviews," Evidence-Based Complementary and Alternative Medicine, vol. 2019, Article ID 7036914, 8 pages, 2019.

[121] N. Milman, T. Paszkowski, I. Cetin, and C. Castelo-Branco, "Supplementation during pregnancy: beliefs and science," Gynecological Endocrinology, vol. 32, no. 7, pp. 509-516, 2016.

[122] E. C. Keats, B. A. Haider, E. Tam, and Z. A. Bhutta, "Multiple-micronutrient supplementation for women during pregnancy," The Cochrane Database of Systematic Reviews, vol. 3, no. 3, Article ID CD004905, 2019.

[123] J. K. Bhardwaj, H. Panchal, and P. Saraf, "Ameliorating effects of natural antioxidant compounds on female infertility: a review," Reproductive Sciences, 2020.

[124] A. Agarwal, A. Aponte-Mellado, B. J. Premkumar, A. Shaman, and S. Gupta, "The effects of oxidative stress on female reproduction: a review," Reproductive Biology and Endocrinology, vol. 10, no. 1, p. 49, 2012.

[125] H. K. Biesalski and J. Tinz, "Multivitamin/mineral supplements: rationale and safety," Nutrition, vol. 36, pp. 60-66, 2017.

[126] K. Turan, A. Arslan, K. Uçkan, H. Demir, and C. Demir, "Change of the levels of trace elements and heavy metals in threatened abortion," Journal of the Chinese Medical Association, vol. 82, no. 7, pp. 554-557, 2019.

[127] R. E. Black, "Micronutrients in pregnancy," British Journal of Nutrition, vol. 85, no. S2, pp. S193-S197, 2001.

[128] P. J. Shen, B. Gong, F. Y. Xu et al., "Four trace elements in pregnant women and their relationships with adverse pregnancy outcomes," European Review for Medical and Pharmacological Sciences, vol. 19, no. 24, pp. 4690-4697, 2015.

[129] L. Prashanth, K. Kattapagari, R. Chitturi et al., "A review on role of essential trace elements in health and disease," Journal of Dr NTR University of Health Sciences, vol. 4, no. 2, pp. 75-85, 2015.

[130] W.-L. Lee, C.-C. Yeh, and P.-H. Wang, "Risk to increase threatened abortion: deficiency of some essential trace elements and exposure of toxic heavy metals," Journal of the Chinese Medical Association, vol. 82, no. 8, pp. 607-608, 2019. 
[131] P. Pathak, U. Kapil, S. N. Dwivedi, and R. Singh, "Serum zinc levels amongst pregnant women in a rural block of Haryana state, India," Asia Pacific Journal of Clinical Nutrition, vol. 17, no. 2, pp. 276-279, 2008.

[132] H. Wang, Y. F. Hu, J. H. Hao et al., "Maternal zinc deficiency during pregnancy elevates the risks of fetal growth restriction: a population-based birth cohort study," Scientific Reports, vol. 5, p. 11262, 2015.

[133] R. Thaker, H. Oza, I. Shaikh, and S. Kumar, "Correlation of copper and zinc in spontaneous abortion," International Journal of Fertility \& Sterility, vol. 13, no. 2, pp. 97-101, 2019.

[134] A. Rumbold, L. Duley, C. A. Crowther et al., "Antioxidants for preventing pre-eclampsia," Cochrane Database of Systematic Reviews, vol. 2008, Article ID CD004227, 2008.

[135] D. Shah and H. P. S. Sachdev, "Zinc deficiency in pregnancy and fetal outcome," Nutrition Reviews, vol. 64, no. 1, pp. 15-30, 2006.

[136] M. Gzhegotskyi and N. Sukhodolska, "Assessment of lead, cadmium, copper and zinc content in women's blood during II trimester of gestation," Experimental and Clinical Physiology and Biochemistry, vol. 2019, no. 2, pp. 5-10, 2019.

[137] M. A. Jankowski-Hennig, M. S. Clegg, G. P. Daston, J. M. Rogers, and C. L. Keen, "Zinc-deficient rat embryos have increased caspase 3-like activity and apoptosis," Biochemical and Biophysical Research Communications, vol. 271, no. 1, pp. 250-256, 2000.

[138] W. R. Ward and D. Bryce-Smith, "Placental element levels in relation to fetal development for obstetrically normal births: a study of 37 elements. Evidence for effects of cadmium, lead and zinc, on fetal growth, and smoking as a source of cadmium," Journal of Biosocial Science, vol. 9, pp. 63-81, 1987.

[139] B. de Benoist, I. Darnton-Hill, L. Davidsson, O. Fontaine, and C. Hotz, "Conclusions of the joint WHO/UNICEF/ IAEA/IZiNCG interagency meeting on zinc status indicators," Food and Nutrition Bulletin, vol. 28, no. 3, pp. S480-S484, 2007.

[140] J. D. Hamadani, G. J. Fuchs, S. J. Osendarp, S. N. Huda, and S. M. Grantham-McGregor, "Zinc supplementation during pregnancy and effects on mental development and behaviour of infants: a follow-up study," The Lancet, vol. 360, no. 9329, pp. 290-294, 2002.

[141] A. Langley and C. T. Dameron, "Copper and anesthesia: clinical relevance and management of copper related disorders," Anesthesiology Research and Practice, vol. 2013, Article ID 750901, 10 pages, 2013.

[142] A. Alebic-Juretic and A. Frkovic, "Plasma copper concentrations in pathological pregnancies," Journal of Trace Elements in Medicine and Biology, vol. 19, no. 2-3, pp. 191-194, 2005.

[143] M. Gzhegotskyi and N. Sukhodolska, "Lead, cadmium, copper and zinc content in women's blood during the third trimester of uncomplicated and complicated gestation," Experimental and Clinical Physiology and Biochemistry, vol. 2019, no. 3, pp. 5-11, 2019.

[144] M. Speich, "Copper in the normal and diabetic pregnancy and in the perinatal period," Revue Française de Gynécologie et d'Obstétrique, vol. 85, no. 1, pp. 34-39, 1990.

[145] P. Borella, A. Szilagyi, G. Than et al., "Maternal plasma concentrations of magnesium, calcium, zinc and copper in normal and pathological pregnancies," Science of the Total Environment, vol. 99, no. 1-2, pp. 67-76, 1990.

[146] Z. Serdar, E. Gür, and O. Develioğlu, "Serum iron and copper status and oxidative stress in severe and mild preeclampsia," Cell Biochemistry and Function, vol. 24, no. 3, pp. 209-215, 2006.

[147] U. Kapil and A. S. Bhadoria, "National iron-plus initiative guidelines for control of iron deficiency anaemia in India, 2013," The National Medical Journal of India, vol. 27, no. 1, pp. 27-29, 2014.

[148] S. Iqbal, P. Rust, L. Weitensfelder et al., "Iron and iodine status in pregnant women from a developing country and its relation to pregnancy outcomes," International Journal of Environmental Research and Public Health, vol. 16, no. 22, p. $4414,2019$.

[149] Y. Guo, N. Zhang, D. Zhang et al., "Iron homeostasis in pregnancy and spontaneous abortion," American Journal of Hematology, vol. 94, no. 2, pp. 184-188, 2019.

[150] J. L. Beard, "Effectiveness and strategies of iron supplementation during pregnancy," The American Journal of Clinical Nutrition, vol. 71, no. 5, pp. 1288S-1294S, 2000.

[151] S. Pavord, B. Myers, S. Robinson, S. Allard, J. Strong, and C. Oppenheimer, "UK guidelines on the management of iron deficiency in pregnancy," British Journal of Haematology, vol. 156, no. 5, pp. 588-600, 2012.

[152] A. F. Goddard, M. W. James, A. S. McIntyre, and B. B. Scott, "Guidelines for the management of iron deficiency anaemia," Gut, vol. 60, no. 10, pp. 1309-1316, 2011.

[153] BC Guidelines, "Iron deficiency-diagnosis and management," 2019, https://www2.gov.bc.ca/gov/content/health/ practitioner-professional-resources/bc-guidelines/irondeficiency.

[154] M. Jariwala, S. Suvarna, G. Kiran Kumar, A. Amin, and A. C. Udas, "Study of the concentration of trace elements Fe, $\mathrm{Zn}, \mathrm{Cu}, \mathrm{Se}$ and their correlation in maternal serum, cord serum and colostrums," Indian Journal of Clinical Biochemistry, vol. 29, no. 2, pp. 181-188, 2014.

[155] O. O. Ajayi, M. A. Charles-Davies, and O. G. Arinola, "Progesterone, selected heavy metals and micronutrients in pregnant Nigerian women with a history of recurrent spontaneous abortion," African Health Sciences, vol. 12, no. 2, pp. 153-159, 2012.

[156] W. J. Omeljaniuk, K. Socha, M. H. Borawska et al., "Antioxidant status in women who have had a miscarriage," Advances in Medical Sciences, vol. 60, no. 2, pp. 329-334, 2015.

[157] N. Hovdenak and K. Haram, "Influence of mineral and vitamin supplements on pregnancy outcome," European Journal of Obstetrics \& Gynecology and Reproductive Biology, vol. 164, no. 2, pp. 127-132, 2012.

[158] E. Mesdaghinia, B. Mohammad-Ebrahimi, F. Foroozanfard, and H. R. Banafshe, "The effect of vitamin E and aspirin on the uterine artery blood flow in women with recurrent abortion: a single-blind randomized controlled trial," International Journal of Reproductive BioMedicine, vol. 15, no. 10, pp. 635-640, 2017.

[159] P. Christoph, P. Challande, L. Raio, and D. Surbek, "High prevalence of severe vitamin $\mathrm{D}$ deficiency during the first trimester in pregnant women in Switzerland and its potential contributions to adverse outcomes in the pregnancy," Swiss Medical Weekly, vol. 150, Article ID w20238, 2020.

[160] S. Bastos Maia, A. S. Rolland Souza, M. F. Costa Caminha et al., "Vitamin A and pregnancy: a narrative review," $\mathrm{Nu}$ trients, vol. 11, no. 3, 2019.

[161] M. E. McCauley, N. van den Broek, L. Dou et al., "Vitamin A supplementation during pregnancy for maternal and newborn outcomes," Cochrane Database of Systematic Reviews, vol. 2015, Article ID CD008666, 2015. 
[162] W. M. Hague, "Homocysteine and pregnancy," Best Practice \& Research Clinical Obstetrics \& Gynaecology, vol. 17, no. 3, pp. 459-469, 2003.

[163] W. Nelen, H. J. Blom, E. A. Steegers et al., "Homocysteine and folate levels as risk factors for recurrent early pregnancy loss," Obstetrics \& Gynecology, vol. 95, no. 4, pp. 519-524, 2000.

[164] A. G. Ronnenberg, S. A. Venners, X. Xu et al., "Preconception B-vitamin and homocysteine status, conception, and early pregnancy loss," American Journal of Epidemiology, vol. 166, no. 3, pp. 304-312, 2007.

[165] M. F. McCarty, "Prenatal high-dose pyridoxine may prevent hypertension and syndrome $\mathrm{X}$ in-utero by protecting the fetus from excess glucocorticoid activity," Medical Hypotheses, vol. 54, no. 5, pp. 808-813, 2000.

[166] J. Shen, C.-Q. Lai, J. Mattei, J. M. Ordovas, and K. L. Tucker, "Association of vitamin B-6 status with inflammation, oxidative stress, and chronic inflammatory conditions: the Boston Puerto Rican health study," The American Journal of Clinical Nutrition, vol. 91, no. 2, pp. 337-342, 2010.

[167] L. R. Solomon and K. Cohen, "Erythrocyte $\mathrm{O}_{2}$ transport and metabolism and effects of vitamin B6 therapy in type II diabetes mellitus," Diabetes, vol. 38, no. 7, pp. 881-886, 1989.

[168] U. Hübner, A. Alwan, M. Jouma et al., "Low serum vitamin B12 is associated with recurrent pregnancy loss in Syrian women," Clinical Chemistry and Laboratory Medicine, vol. 46, no. 9, pp. 1265-1269, 2008.

[169] E. M. Guerra-Shinohara, P. M. Pereira, A. M. Kubota et al., "Increased MMA concentration and body mass index are associated with spontaneous abortion in Brazilian women: a pilot study," Clinica Chimica Acta, vol. 411, no. 5-6, pp. 423-427, 2010.

[170] M. F. Reznikoff-Etiévant, J. Zittoun, C. Vaylet, P. Pernet, and J. Milliez, "Low Vitamin B12 level as a risk factor for very early recurrent abortion," European Journal of Obstetrics \& Gynecology and Reproductive Biology, vol. 104, no. 2, pp. 156-159, 2002.

[171] H. Cuny, M. Rapadas, J. Gereis et al., "NAD deficiency due to environmental factors or gene-environment interactions causes congenital malformations and miscarriage in mice," Proceedings of the National Academy of Sciences, vol. 117, no. 7, pp. 3738-3747, 2020.

[172] F. Li, T. Fushima, G. Oyanagi et al., "Nicotinamide benefits both mothers and pups in two contrasting mouse models of preeclampsia," Proceedings of the National Academy of Sciences, vol. 113, no. 47, pp. 13450-13455, 2016.

[173] U.S.P.S.T. Force, "Folic acid for the prevention of neural tube defects: U.S. preventive services task force recommendation statement," Annals of Internal Medicine, vol. 150, no. 9, pp. 626-631, 2009.

[174] A. M. Cordero, K. S. Crider, L. M. Rogers, M. J. Cannon, and R. J. Berry, "Optimal serum and red blood cell folate concentrations in women of reproductive age for prevention of neural tube defects: World Health Organization guidelines," MMWR. Morbidity and Mortality Weekly Report, vol. 64, no. 15, pp. 421-423, 2015.

[175] ACoP Bulletins, "ACOG practice bulletin. Clinical management guidelines for obstetrician-gynecologists," Obstetrics \& Gynecology, vol. 102, no. 1, pp. 203-213, 2003.

[176] R. D. Wilson, G. Davies, V. Désilets et al., "The use of folic acid for the prevention of neural tube defects and other congenital anomalies," Journal of Obstetrics and Gynaecology Canada, vol. 25, no. 11, pp. 959-973, 2003.
[177] K. Bibbins-Domingo, D. C. Grossman, S. J. Curry et al., "Folic acid supplementation for the prevention of neural tube defects: US preventive services task force recommendation statement," The Journal of the American Medical Association, vol. 317, no. 2, pp. 183-189, 2017.

[178] L. George, J. L. Mills, A. L. Johansson et al., "Plasma folate levels and risk of spontaneous abortion," JAMA, vol. 288, no. 15, pp. 1867-1873, 2002.

[179] N. Maconochie, P. Doyle, S. Prior, and R. Simmons, "Risk factors for first trimester miscarriage-results from a UKpopulation-based case-control study," BJOG: An International Journal of Obstetrics \& Gynaecology, vol. 114, no. 2, pp. 170-186, 2007.

[180] S. Brody, "High-dose ascorbic acid increases intercourse frequency and improves mood: a randomized controlled clinical trial," Biological Psychiatry, vol. 52, no. 4, pp. 371-374, 2002.

[181] S. R. Al-Katib, M. M. Al-Kaabi, and K. Al-Jashamy, "Effects of vitamin $\mathrm{C}$ on the endometrial thickness and ovarian hormones of progesterone and estrogen in married and unmarried women," American Journal of Research Communication, vol. 1, no. 8, pp. 24-31, 2013.

[182] N. Di Marco, J. Kaufman, and C. P. Rodda, "Shedding light on vitamin $\mathrm{D}$ status and its complexities during pregnancy, infancy and childhood: an Australian perspective," International Journal of Environmental Research and Public Health, vol. 16, no. 4, 2019.

[183] M. F. Holick, "Vitamin D status: measurement, interpretation, and clinical application," Annals of Epidemiology, vol. 19, no. 2, pp. 73-78, 2009.

[184] A. Mousa, M. Misso, H. Teede et al., "Effect of vitamin D supplementation on inflammation: protocol for a systematic review," British Medical Journal Open, vol. 6, no. 4, Article ID e010804, 2016.

[185] M. F. Holick, "Vitamin D deficiency in 2010: health benefits of vitamin D and sunlight: a D-bate," Nature Reviews Endocrinology, vol. 7, no. 2, pp. 73-75, 2011.

[186] A. Prentice, "Vitamin D deficiency: a global perspective," Nutrition Reviews, vol. 66, no. 10, pp. S153-S164, 2008.

[187] R. M. Daly, C. Gagnon, Z. X. Lu et al., "Prevalence of vitamin $\mathrm{D}$ deficiency and its determinants in Australian adults aged 25 years and older: a national, population-based study," Clinical Endocrinology, vol. 77, no. 1, pp. 26-35, 2012.

[188] T. K. Gill, C. L. Hill, E. M. Shanahan et al., "Vitamin D levels in an Australian population," BMC Public Health, vol. 14, p. 1001, 2014.

[189] E. Moghadas Inanloo, Z. Keshavarz, Z. Naeiji et al., "Comparison of serum vitamin D levels in threatened abortion patients and women with normal pregnancy," The Iranian Journal of Obstetrics, Gynecology and Infertility, vol. 22, no. 10, pp. 49-57, 2019.

[190] L. B. Andersen, J. S. Jorgensen, T. K. Jensen et al., "Vitamin D insufficiency is associated with increased risk of first-trimester miscarriage in the Odense child cohort," American Journal of Clinical Nutrition, vol. 102, no. 3, pp. 633-638, 2015.

[191] I. K. M. Bakleicheva and O. Bespalova, "The role of vitamin D for threatened miscarriage in the first trimester of pregnancy," in Proceedings of the 18th World Congress Gynecological Endocrinology, Florence, Italy, 2018.

[192] J.-C. Souberbielle, "Epidemiology of vitamin-D deficiency," Gériatrie et Psychologie Neuropsychiatrie du Viellissement, vol. 14, no. 1, pp. 7-15, 2016. 
[193] N. R. Parva, S. Tadepalli, P. Singh et al., "Prevalence of vitamin D deficiency and associated risk factors in the US population (2011-2012)," The Cureus Journal of Medical Science, vol. 10, no. 6, p. e2741, 2018.

[194] M. Fernando, S. J. Ellery, C. Marquina, S. Lim, N. Naderpoor, and A. Mousa, "Vitamin D-binding protein in pregnancy and reproductive health," Nutrients, vol. 12, no. 5, p. 1489, 2020.

[195] H. Hou, J. Y. Zhang, D. Chen et al., "Altered decidual and placental catabolism of vitamin D may contribute to the aetiology of spontaneous miscarriage," Placenta, vol. 92, pp. 1-8, 2020.

[196] M. Samimi, F. Foroozanfard, F. Amini et al., "Effect of vitamin D supplementation on unexplained recurrent spontaneous abortion: a double-blind randomized controlled trial," Global Journal of Health Science, vol. 9, no. 3, p. 57723, 2016.

[197] L. Díaz, N. Noyola-Martínez, D. Barrera et al., "Calcitriol inhibits TNF- $\alpha$-induced inflammatory cytokines in human trophoblasts," Journal of Reproductive Immunology, vol. 81, no. 1, pp. 17-24, 2009.

[198] I. Bubanovic, "1 $\alpha, 25$-dihydroxy-vitamin-D3 as new immunotherapy in treatment of recurrent spontaneous abortion," Medical Hypotheses, vol. 63, no. 2, pp. 250-253, 2004.

[199] A. Mithal and S. Kalra, "Vitamin D supplementation in pregnancy," Indian Journal of Endocrinology and Metabolism, vol. 18, no. 5, pp. 593-596, 2014.

[200] P. Vural, C. Akgül, A. Yildirim et al., "Antioxidant defence in recurrent abortion," Clinica Chimica Acta, vol. 295, no. 1-2, pp. 169-177, 2000.

[201] A. A. Shamim, K. Schulze, R. D. Merrill et al., "First-trimester plasma tocopherols are associated with risk of miscarriage in rural Bangladesh," The American Journal of Clinical Nutrition, vol. 101, no. 2, pp. 294-301, 2015.

[202] L. Bártfai, Z. Bártfai, I. Nedeczky, E. H. Puho, F. Bánhidy, and A. E. Czeizel, "Rate of preterm birth in pregnant women with vitamin E treatment: a population-based study," The Journal of Maternal-Fetal \& Neonatal Medicine, vol. 25, no. 6, pp. 575-580, 2012.

[203] J. A. Spinnato II, S. Freire, J. L. Pinto e Silva et al., "Antioxidant supplementation and premature rupture of the membranes: a planned secondary analysis," American Journal of Obstetrics and Gynecology, vol. 199, no. 4, p. 433.e1, 2008.

[204] M. Szilasi, L. Bártfai, Z. Bártfai, F. Bánhidy, and A. E. Czeizel, "No association of maternal vitamin $\mathrm{E}$ intake with higher risk of cardiovascular malformations in children: a population-based case-control study," Therapeutic Advances in Drug Safety, vol. 2, no. 3, pp. 77-86, 2011.

[205] H. K. Biesalski and J. Tinz, "Multivitamin/mineral supplements: rationale and safety-a systematic review," Nutrition, vol. 33, pp. 76-82, 2017.

[206] S. Gupta, A. Agarwal, J. Banerjee, and J. G. Alvarez, "The role of oxidative stress in spontaneous abortion and recurrent pregnancy loss: a systematic review," Obstetrical \& Gynecological Survey, vol. 62, no. 5, pp. 335-347, 2007.

[207] M. G. Showell, R. Mackenzie-Proctor, V. Jordan, and R. J. Hart, "Antioxidants for female subfertility," The Cochrane Database of Systematic Reviews, vol. 7, no. 7, Article ID CD007807, 2017.

[208] C. Di Tucci, M. Di Feliciantonio, F. Vena et al., "Alpha lipoic acid in obstetrics and gynecology," Gynecological Endocrinology, vol. 34, no. 9, pp. 729-733, 2018.
[209] G. Monastra, S. De Grazia, S. Cilaker Micili, A. Goker, and V. Unfer, "Immunomodulatory activities of alpha lipoic acid with a special focus on its efficacy in preventing miscarriage," Expert Opinion on Drug Delivery, vol. 13, no. 12, pp. 16951708, 2016.

[210] S. C. Micili, A. Goker, O. Sayin, P. Akokay, and B. U. Ergur, "The effect of lipoic acid on wound healing in a full thickness uterine injury model in rats," Journal of Molecular Histology, vol. 44, no. 3, pp. 339-345, 2013.

[211] P. Bao, A. Kodra, M. Tomic-Canic, M. S. Golinko, H. P. Ehrlich, and H. Brem, "The role of vascular endothelial growth factor in wound healing," Journal of Surgical Research, vol. 153, no. 2, pp. 347-358, 2009.

[212] H. Ma and S. Cherng, "Alpha-smooth muscle actin (alphaSMA)," The Journal of American Science, vol. 4, no. 2, pp. 1-3, 2008.

[213] A. Talebi, S. Zavareh, M. H. Kashani, T. Lashgarbluki, and I. Karimi, "The effect of alpha lipoic acid on the developmental competence of mouse isolated preantral follicles," Journal of Assisted Reproduction and Genetics, vol. 29, no. 2, pp. 175-183, 2012.

[214] G. Porcaro, E. Brillo, I. Giardina et al., "Alpha lipoic acid (ALA) effects on subchorionic hematoma: preliminary clinical results," European Review for Medical and Pharmacological Sciences, vol. 19, no. 18, pp. 3426-3432, 2015.

[215] M. Costantino, C. Guaraldi, and D. Costantino, "Resolution of subchorionic hematoma and symptoms of threatened miscarriage using vaginal alpha lipoic acid or progesterone: clinical evidences," European Review for Medical and Pharmacological Sciences, vol. 20, no. 8, pp. 1656-1663, 2016.

[216] A. Goraca, H. Huk-Kolega, A. Piechota et al., "Lipoic acid-biological activity and therapeutic potential," Pharmacological Reports, vol. 63, no. 4, pp. 849-858, 2011.

[217] A. Fulghesu, M. Ciampelli, G. Muzj et al., "N-acetyl-cysteine treatment improves insulin sensitivity in women with polycystic ovary syndrome," Fertility and Sterility, vol. 77, no. 6, pp. 1128-1135, 2002.

[218] D. Thakker, A. Raval, I. Patel et al., "N-acetylcysteine for polycystic ovary syndrome: a systematic review and metaanalysis of randomized controlled clinical trials," Obstetrics and Gynecology International, vol. 2015, Article ID 817849, 13 pages, 2015.

[219] A. Nasr, "Effect of N-acetyl-cysteine after ovarian drilling in clomiphene citrate-resistant PCOS women: a pilot study," Reproductive BioMedicine Online, vol. 20, no. 3, pp. 403-409, 2010.

[220] H. Abu Hashim, K. Anwar, and R. A. El-Fatah, "N-acetyl cysteine plus clomiphene citrate versus metformin and clomiphene citrate in treatment of clomiphene-resistant polycystic ovary syndrome: a randomized controlled trial," Journal of Women's Health, vol. 19, no. 11, pp. 2043-2048, 2010.

[221] Y. Zhang, W. Zhao, H. Xu et al., "Hyperandrogenism and insulin resistance-induced fetal loss: evidence for placental mitochondrial abnormalities and elevated reactive oxygen species production in pregnant rats that mimic the clinical features of polycystic ovary syndrome," The Journal of Physiology, vol. 597, no. 15, pp. 3927-3950, 2019.

[222] N. Lazzarin, E. Vaquero, C. Exacoustos, E. Bertonotti, M. E. Romanini, and D. Arduini, "Low-dose aspirin and omega-3 fatty acids improve uterine artery blood flow velocity in women with recurrent miscarriage due to impaired uterine perfusion," Fertility and Sterility, vol. 92, no. 1, pp. 296-300, 2009. 
[223] L. A. Wise, A. K. Wesselink, K. L. Tucker et al., "Dietary fat intake and fecundability in 2 preconception cohort studies," American Journal of Epidemiology, vol. 187, no. 1, pp. 60-74, 2018.

[224] Y.-H. Chiu, A. E. Karmon, A. J. Gaskins et al., "Serum omega-3 fatty acids and treatment outcomes among women undergoing assisted reproduction," Human Reproduction, vol. 33, no. 1, pp. 156-165, 2018.

[225] J. Farren, N. Mitchell-Jones, J. Y. Verbakel, D. Timmerman, M. Jalmbrant, and T. Bourne, "The psychological impact of early pregnancy loss," Human Reproduction Update, vol. 24, no. 6, pp. 731-749, 2018.

[226] P. P. Karaca and Ü. Y. Oskay, "Effect of supportive care on the psychosocial health status of women who had a miscarriage," Perspectives in Psychiatric Care, vol. 57, no. 1, pp. 179-188, 2021.

[227] K. Conway and G. Russell, "Couples' grief and experience of support in the aftermath of miscarriage," British Journal of Medical Psychology, vol. 73, no. 4, pp. 531-545, 2000.

[228] M. Frost and J. T. Condon, "The psychological sequelae of miscarriage: a critical review of the literature," Australian \& New Zealand Journal of Psychiatry, vol. 30, no. 1, pp. 54-62, 1996.

[229] J. E. Bilardi, G. Sharp, S. Payne, and M. J. Temple-Smith, "The need for improved emotional support: a pilot online survey of Australian women's access to healthcare services and support at the time of miscarriage," Women and Birth, 2020.

[230] F. McCarthy, R. Moss-Morris, A. Khashan et al., "Previous pregnancy loss has an adverse impact on distress and behaviour in subsequent pregnancy," BJOG: An International Journal of Obstetrics \& Gynaecology, vol. 122, no. 13, pp. 1757-1764, 2015.

[231] H. R. Hall and K. Jolly, "Women's use of complementary and alternative medicines during pregnancy: a cross-sectional study," Midwifery, vol. 30, no. 5, pp. 499-505, 2014.

[232] M. Kalder, K. Knoblauch, I. Hrgovic, and K. Münstedt, "Use of complementary and alternative medicine during pregnancy and delivery," Archives of Gynecology and Obstetrics, vol. 283, no. 3, pp. 475-482, 2011.

[233] S. Bershadsky, L. Trumpfheller, H. B. Kimble, D. Pipaloff, and I. S. Yim, "The effect of prenatal Hatha yoga on affect, cortisol and depressive symptoms," Complementary Therapies in Clinical Practice, vol. 20, no. 2, pp. 106-113, 2014.

[234] J. Huberty, J. A. Leiferman, A. R. Kruper et al., "Exploring the need for interventions to manage weight and stress during interconception," Journal of Behavioral Medicine, vol. 40, no. 1, pp. 145-158, 2017.

[235] H. H. Ho, J. F. O'Connor, S. T. Nakajima, J. Tieu, J. W. Overstreet, and B. L. Lasley, "Characterization of human chorionic gonadotropin in normal and abnormal pregnancies," Early Pregnancy: Biology and Medicine: The Official Journal of the Society for the Investigation of Early Pregnancy, vol. 3, no. 3, pp. 213-224, 1997.

[236] P. Devaseelan, P. P. Fogarty, and L. Regan, "Human chorionic gonadotrophin for threatened miscarriage," Cochrane Database Systematic Reviews, vol. 12, no. 5, Article ID CD007422, 2010. 